\title{
A COMPLEX HOMOGRAPHIC BEST APPROXIMATION PROBLEM. APPLICATION TO OPTIMIZED ROBIN-SCHWARZ ALGORITHMS, AND OPTIMAL CONTROL PROBLEMS
}

\author{
BÉRANGÈRE DELOURME* AND LAURENCE HALPERN ${ }^{\dagger}$
}

\begin{abstract}
Homographic complex best approximation has emerged in the last years, as an essential tool for the design of new, performant domain decomposition Robin-Schwarz algorithms. We present and analyse a fully complex problem, introducing a new alternation property. We give operational formulas for the solution, and apply them to a control problem.
\end{abstract}

AMS subject classifications. 30E10; 41A10; 41A50; 65K10; 49M27; 65N55; 90C47

Key words. Complex best approximation; Domain decomposition; Schwarz algorithms; Optimal control.

1. Introduction. Optimized Schwarz algorithms are recent tools for domain decomposition in view of parallelization. They use more efficient and flexible transmission conditions than the classical Schwarz algorithm, which needs overlapping subdomains, and uses Dirichlet transmission only. In the third paper of his seminal series, see [32], P.L. Lions opened the door to the use of new transmission conditions without overlap: At each step, we solve the same equation in each subdomain "passing from each subdomain to the others a convex combination of Neumann and Dirichlet data": in particular this yields a Robin (or Fourier) type boundary condition on each interface. B. Després in his thesis used radiation transmission conditions [2] on the interface for Helmholtz equation [12]. T. Hagström and collaborators presented in [22] the first numerical experiments with "optimal" transmission conditions: For rectangular domains and separable linear operators, optimal choices of the boundary conditions can be made. F. Nataf and cooauthors gave an extended analysis of the optimal transmission conditions, see [36]. Since then optimized transmission conditions have been designed, starting in C. Japhet's thesis, see $[28,14]$. The principle at the root of optimized Robin-Schwarz methods is to find the coefficients in the Robin transmission conditions which optimize the convergence factor of the algorithm. This is achieved in the most simple case of two half-spaces or rectangular subdomains by using a Fourier transform or Fourier series in the direction of the interface. The convergence factor can then be calculated in closed form, as a function of the parameter $\ell$ in the Robin transmission condition, and the frequency $k$. For the equation $-\Delta u+\eta u=0$, with $\eta \in \mathbb{C} \backslash \mathbb{R}_{-}$, define the function

$$
\delta_{L}(\ell, k)=\left|\frac{\omega(k)-\ell}{\omega(k)+\ell} e^{-L \omega(k)}\right|, \text { with } \omega(k)=\sqrt{k^{2}+\eta} .
$$

$L \geq 0$ is the width of the overlap, $K$ is a closed interval

$$
K=\left[k_{\min }, k_{\max }\right],
$$

with $k_{\max } \in \overline{\mathbb{R}}$ when $L>0$. The determination of the best parameter $\ell$ is a min-max problem: to find $\left(\ell_{L}^{*}, \delta_{L}^{*}\right)$ such that

$$
\delta_{L}^{*}=\sup _{k \in K} \delta_{L}\left(\ell_{L}^{*}, k\right)=\inf _{\ell \in \mathbb{C}} \sup _{k \in K} \delta_{L}(\ell, k) .
$$

*Université Sorbonne Paris Nord, LAGA, Villetaneuse, France, delourme@math.univ-paris13.fr

${ }^{\dagger}$ Université Sorbonne Paris Nord, LAGA, Villetaneuse, France, halpern@math.univ-paris13.fr 
Problem (1.3) can be seen as a best approximation problem by polynomials of degree 0 .

Historically, the first best approximation problem on $\mathbf{P}_{n}(\mathbb{C})$ (complex polynomials of degree less than or equal to $n)$, starts with the linear problem

$$
\inf _{P \in \mathbf{P}_{n}(\mathbb{C})} \sup _{z \in K} \zeta(P, z), \quad \zeta(P, z)=|f(z)-P(z)| .
$$

For a continuous function $f$, this problem has a unique solution, see for instance the book by Meinardus [35]. The alternation theorem, due to Chebyshev (necessary condition) and De la Vallée Poussin (sufficient condition) in the real case, is probably due to Tonelli [43] and extended by Rivlin and Shapiro [37], see [42]. For any $P \in$ $\mathbf{P}_{n}(\mathbb{C})$, let $E$ be the set of points $z \in K$ such that $|f(z)-P(z)|=\|f-P\|_{L^{\infty}(K)}$.

Theorem 1.1 (Rivlin-Shapiro [37]). A polynomial $P$ is a polynomial of linear best approximation for $f \in \mathcal{C}(K)$ if and only if there are $r$ points $z_{1}, \cdots, z_{r} \in E$ (alternation points) and $r$ numbers $p_{1}, \cdots, p_{r}>0(r \leq 2 n+3$ in the complex case and $r \leq n+2$ in the real case) for which

$$
\sum_{j=1}^{r} p_{j}\left(f\left(z_{j}\right)-P\left(z_{j}\right)\right) \overline{\phi_{i}\left(z_{j}\right)}=0, \quad i=1, \cdots, n+1,
$$

where the $\phi_{i}$ are a basis of the space $\mathbf{P}_{n}(\mathbb{C})$. In the real case, there are exactly $n+2$ alternation points.

The previous theorem has been extended to the weighted homographic best approximation problem in [7],

$$
\inf _{P \in \mathbf{P}_{n}(\mathbb{C})} \sup _{z \in K} \zeta(P, z), \quad \zeta(P, z)=\left|\frac{f(z)-P(z)}{f(z)+P(z)} e^{-L f(z)}\right|,
$$

in the context of the optimized Schwarz method for the advection-diffusion equation. That case is symmetric, therefore the best coefficient is real. The present paper adapts and extends those results for the Robin-Schwarz algorithm (corresponding to $n=0$ in (1.6)) to the fully complex problem. The method of investigation follows the approach presented in [7]. We list below the main steps, as well as the associated theorems, which are proven in the core of the article. A short preliminary account for this analysis has been given in [11].

\section{Roadmap and main results.}

1.A Well-posedness and equioscillation (Theorems 2.4-2.5-2.8) .

For any $L>0$ and $k_{\max } \in \overline{\mathbb{R}}$, or for $L=0$ and any $k_{\max } \in \mathbb{R}$, there is a unique $\left(\delta_{L}^{*}, \ell_{L}^{*}\right)$ solution of (1.3).

Furthermore there are at least two equioscillation points for $\ell_{L}^{*}$ : there are $k_{1}$ and $k_{2}$ distinct in $K$ such that

$$
\delta_{L}^{*}=\delta_{L}\left(\ell_{L}^{*}, k_{1}\right)=\delta_{L}\left(\ell_{L}^{*}, k_{2}\right) .
$$

\section{B Alternation (Theorem and Definition 3.2).}

Let $\hat{\ell} \in \mathbb{C}$ such that there are two alternating points $k_{j}$ in $K$, that is

$$
\begin{gathered}
\delta_{L}\left(\hat{\ell}, k_{1}\right)=\delta_{L}\left(\hat{\ell}, k_{2}\right)=\sup _{k \in K} \delta_{L}(\hat{\ell}, k), \\
\exists p \in \mathbb{R}_{+}^{*}, \nabla_{\ell} \delta_{L}\left(\hat{\ell}, k_{2}\right)+p \nabla_{\ell} \delta_{L}\left(\hat{\ell}, k_{1}\right)=0 .
\end{gathered}
$$


Then $\hat{\ell}=\ell_{L}^{*}$.

1.C Operational formulas: non-overlapping case (Theorem 3.7). Define

$$
\omega_{\min }=\omega\left(k_{\min }\right), \omega_{\max }=\omega\left(k_{\max }\right), \theta_{\min }=\operatorname{Arg} \omega_{\min }, \theta_{\max }=\operatorname{Arg} \omega_{\max },
$$

where $\omega$ is defined in (1.1).

Assume that $L=0$ and $k_{\max } \in \mathbb{R}$.

1. If $\theta_{\min } \leq \frac{\pi}{4}$, or if $\frac{\pi}{4}<\theta_{\min }<\frac{\pi}{3}$ and $k_{\max }$ is sufficiently large, then

$$
\ell_{0}^{*}=\sqrt{\omega_{\min } \omega_{\max }}, \quad \delta_{0}^{*}=\left|\frac{\sqrt{\omega_{\max }}-\sqrt{\omega_{\min }}}{\sqrt{\omega_{\max }}+\sqrt{\omega_{\min }}}\right| .
$$

2. If $\theta_{\min } \geq \frac{\pi}{3}$ and $k_{\max }$ is sufficiently large, then

$$
\ell_{0}^{*} \sim \sqrt{\sqrt{\frac{2 \operatorname{Im} \eta}{\sqrt{3}}} \omega_{\max }} e^{i \frac{\pi}{6}}, \quad \delta_{0}^{*} \sim 1-\sqrt{\frac{\sqrt{6 \sqrt{3} \operatorname{Im} \eta}}{k_{\max }}} .
$$

1.D Operational formulas: overlapping case (Theorem 3.14 and Theorem 3.17).

Suppose $k_{\max }=+\infty$. Then for $L>0$ sufficiently small, the optimal parameter $\ell_{L, \infty}^{*}$ and the corresponding convergence factor $\delta_{L, \infty}^{*}$ admit the following asymptotic expansions:

1. If $\theta_{\min } \leq \frac{\pi}{3}$,

$$
\ell_{L, \infty}^{*} \sim\left(\frac{\left|\omega_{\min }\right|^{2}}{2 L} \cos \frac{\theta_{\min }}{2}\right)^{\frac{1}{3}} e^{i \frac{\theta_{\min }}{2}}, \delta_{L, \infty}^{*} \sim 1-2 \sqrt{2 L \operatorname{Re} \ell_{L, \infty}^{*}}
$$

2. If $\theta_{\min }>\frac{\pi}{3}$,

$$
\ell_{L, \infty}^{*} \sim\left(\frac{\operatorname{Im} \eta}{2 L}\right)^{\frac{1}{3}} e^{i \frac{\pi}{6}}, \quad \delta_{L, \infty}^{*} \sim 1-2 \sqrt{2 L \operatorname{Re} \ell_{L, \infty}^{*}} .
$$

If $k_{\max } \in \mathbb{R}$ is sufficiently large, the formulas are still valid.

Well-posedness is proved without any constrains on the overlap $L$. A new necessary and sufficient condition is given based on alternation of the derivative in $\ell$, see property 1.B above.

Context and outline. Many authors have analyzed optimized Schwarz algorithms, the first one [16] concerns the real case $\left(\eta \in \mathbb{R}_{+}\right)$for $n=0$ in (1.4) (Robin) and $n=1$ (Ventcel). One can cite also the Helmholtz equation in [15], [24] for the Schrödinger equation. For more complicated problems, such as steady advectiondiffusion with discontinuous coefficients [17], Helmholtz equation with two-sided Robin conditions [20], asymptotically best coefficients with respect to the maximum frequency in the discretized problem were computed by a heuristic equioscillation principle. Drastic improvements using this strategy have been validated by computations, even for non constant coefficients, more general decomposition in subdomains, nonlinear problems, applying the best coefficient locally see [6, 23, 9]. They have been used successfully in connection with asynchronous algorithms [33].

The need for a new fully complex analysis appears in various settings, ranging from physics to numerics. The d'Alembert equation is a model for the displacement of 
vibrating membranes or the components of the electric field in the Maxwell equations. The computation of harmonic solutions with frequency $\kappa$ leads to the Helmholtz equation, that is $\eta=-\frac{\kappa^{2}}{c^{2}}$. In the frame of Ohm's law in an electric circuit, the electric field is proportional to the current density, thus leading to $\Delta u+\left(\frac{\kappa^{2}}{c^{2}}+i \kappa \sigma\right) u=0$, where $\sigma$ is the conductivity. A small imaginary part is also often used to actually compute the solution of the Helmholtz equation, similar to the limit absorption principle. Finally, a purely imaginary coefficient $\eta=\frac{i}{\sqrt{\nu}}$ appears when solving optimal control problems, in the astute formulation introduced by J.D. Benamou in [3], see Section 4.

Our paper deals with any complex coefficients $\eta \in \mathbb{C} \backslash \mathbb{R}_{-}$and is organized as follows. In Section 2 we introduce the alternate Robin-Schwarz algorithm for two half-pipes, and the min-max problem. Then we prove the property 1.A together with a strict local minimum property, dealing separately with the non-overlapping and overlapping cases. Results for the first case are included in previous results in $[7,6]$, while in the second case we obtain a new result for any size of the overlap parameter $L$ by reducing the min-max problem to a compact set. Section 3 provides a characterization of the best parameter in 1.C and 1.D using the equioscillation property, and introducing an alternation theory. We first prove the new sufficient condition for strict optimum in the alternation property 1.B. Then we provide separately in the cases $L>0$ and $L=0$ the algorithm for defining the value $\ell$ which makes the convergence factor alternate twice. Then we provide exact or asymptotic values. In Section 4, we concentrate on the application to optimal control. We give details on the methods by Benamou and Després for domain decomposition, see [3, 5], and provide numerical evidence of the capability of the method. In Section 5, we describe the problems to which our analysis should extend. For a study of the elliptic control problem see [44]. To help the reader, a glossary (see Table 5.1) containing the references and definitions of the main objects used in the text can be found at the end of the article.

\section{Definition and well-posedness for the best approximation problem.}

2.1. Definition of the alternate Schwarz algorithm. Consider the Helmholtz equation with complex coefficient $\eta \in \mathbb{C} \backslash \mathbb{R}_{-}$in the domain $\Omega \subset \mathbb{R}^{d+1}$. Dirichlet boundary conditions are imposed on the boundary $\partial \Omega$.

$$
-\Delta w+\eta w=g \text { in } \Omega, w=0 \text { on } \partial \Omega, \quad \eta=\alpha+2 i \mu, \alpha \in \mathbb{R} \text { and } \mu>0 .
$$

The domain $\Omega$ is split into two subdomains, with or without overlap, and the alternate Robin-Schwarz algorithm introduced by P.L. Lions in [32] works as follows. An initial guess $w_{2}^{0}$ is given in $\Omega_{2}$. The algorithm computes alternatively in the subdomains $\Omega_{j}$ :

$$
\begin{aligned}
& -\Delta w_{1}^{n}+\eta w_{1}^{n}=g \text { in } \Omega_{1}, w_{1}^{n}=0 \text { on } \partial \Omega_{1} \cap \partial \Omega, \\
& \partial_{n_{1}} w_{1}^{n}+\ell w_{1}^{n}=\partial_{n_{1}} w_{2}^{n-1}+\ell w_{2}^{n-1} \text { on } \Gamma_{1}=\partial \Omega_{1} \cap \overline{\Omega_{2}}, \\
& -\Delta w_{2}^{n}+\eta w_{2}^{n}=g \text { in } \Omega_{2}, w_{2}^{n}=0 \text { on } \partial \Omega_{2} \cap \Omega, \\
& \partial_{n_{2}} w_{2}^{n}+\ell w_{2}^{n}=\partial_{n_{2}} w_{1}^{n}+\ell w_{1}^{n} \text { on } \Gamma_{2}=\partial \Omega_{2} \cap \overline{\Omega_{1}} .
\end{aligned}
$$

The vector $n_{j}$ denotes the outward unit normal vector to $\Gamma_{j}$ and $\partial_{n_{j}}$ is the normal derivative on $\Gamma_{j} . \ell$ is a complex parameter which will be searched so as to optimize the convergence factor of the algorithm. The original Schwarz algorithm in [40], that is usually called classical, exchanges Dirichlet data on the interfaces as

$$
w_{1}^{n}=w_{2}^{n-1} \text { on } \Gamma_{1}, \quad w_{2}^{n}=w_{1}^{n} \text { on } \Gamma_{2},
$$

and requires an overlap. The parallel algorithms are similar, updating $w_{2}^{n}$ with $w_{1}^{n-1}$ on the interface, see [32]. 
2.2. The series expansion and the convergence factor. Here $\Omega=\mathbb{R} \times D$, $\left.D=\prod\right] a_{i}, b_{i}\left[\right.$, the subdomains are $\Omega_{1}=(-\infty, L) \times D$ and $\Omega_{2}=(0,+\infty) \times D$; the interfaces are $\Gamma_{1}=\{L\} \times D, \Gamma_{2}=\{0\} \times D$.

TheOREM 2.1. If $\ell$ belongs to the quarter plane $\mathcal{Q}=\{z \in \mathbb{C}, \operatorname{Arg} z \in] 0, \frac{\pi}{2}[\}$, then for $j=1,2$ the problem defining $w_{j}^{n}$ is well-posed in $H^{1}\left(\Omega_{j}\right)$ and the Robin-Schwarz algorithm is convergent.

Proof. The proof of well-posedness for $\eta \in i \mathbb{R}$ can be found in [5] for instance, and extends without difficulty here. The convergence result sits in the same series of papers by Benamou and Després in the non-overlapping case (with several subdomains). In the overlapping case there is no proof available for a general partition into subdomains, the half-pipe case is treated below, using Fourier series in the transverse variable $\boldsymbol{y}$,

$$
u=\sum_{\boldsymbol{q} \in \mathbb{Z}^{d}} \hat{u}(x, \boldsymbol{q}) \prod_{j=1}^{d} \sin \kappa_{j}\left(y_{j}-a_{j}\right), \quad \kappa_{j}=\frac{\pi q_{j}}{b_{j}-a_{j}} .
$$

The errors after $n$ iterations $e_{j}^{n}=w_{j}^{n}-w$, follow the same algorithm with vanishing righthand side. The Fourier coefficients denoted by $\hat{e}_{j}^{n}(x, \boldsymbol{q})$, satisfy the equation

$$
-\left(\hat{e}_{j}^{n}\right)_{x x}+\left(\|\kappa\|^{2}+\eta\right) \hat{e}_{j}^{n}=0 \quad \text { with } \boldsymbol{\kappa}=\left\{\kappa_{j}\right\},\|\boldsymbol{\kappa}\|^{2}=\sum_{j=1}^{d} \kappa_{j}^{2} .
$$

Since the errors are in $H^{1}\left(\Omega_{j}\right)$, their Fourier coefficients cannot be exponentially increasing in $x$, therefore

$$
\hat{e}_{1}^{n}(x, \boldsymbol{q})=a_{1}^{n}(\boldsymbol{q}) e^{\omega x}, \hat{e}_{2}^{n}(x, \boldsymbol{q})=a_{2}^{n}(\boldsymbol{q}) e^{-\omega x}, \quad \omega=\sqrt{\|\boldsymbol{\kappa}\|^{2}+\eta} .
$$

For $\operatorname{Im} z>0, \sqrt{z}$ is the usual principal branch of the square root of $z$, and since $\operatorname{Im} \eta \neq 0$, the complex square root $\omega$ is perfectly defined in the quarter plane $\mathcal{Q}$.

By the interface conditions, the coefficients $a_{j}^{n}(\boldsymbol{q})$ satisfy the recursion relation (using that $\partial_{n_{1}} \equiv \partial_{x}$ and $\partial_{n_{2}} \equiv-\partial_{x}$ ),

$$
(\omega+\ell) a_{1}^{n}(\boldsymbol{q}) e^{\omega L}=(-\omega+\ell) a_{2}^{n-1}(\boldsymbol{q}) e^{-\omega L}, \quad(\omega+\ell) a_{2}^{n}(\boldsymbol{q})=(-\omega+\ell) a_{1}^{n}(\boldsymbol{q}) .
$$

Therefore for $n \geq 1$,

$$
a_{j}^{n+1}=\left(\frac{\omega-\ell}{\omega+\ell} e^{-\omega L}\right)^{2} a_{j}^{n}=\left(\frac{\omega-\ell}{\omega+\ell} e^{-\omega L}\right)^{2 n} a_{j}^{1} .
$$

Define $k=\|\boldsymbol{\kappa}\|, \omega(k)$ and $\delta_{L}(\ell, k)$ from $(1.1,1.3)$. Then for $j=1,2$, for $n \geq 1$,

$$
\left|\hat{e}_{j}^{n}(x, \boldsymbol{q})\right|=\left(\delta_{L}(\ell, k)\right)^{2 n}\left|\hat{e}_{j}^{1}(x, \boldsymbol{q})\right| .
$$

For $\omega$ and $\ell$ in the quarter plane $\mathcal{Q}, \operatorname{Re} \omega \bar{\ell}>0$. The complex identity

$$
\left|z_{1}+z_{2}\right|^{2}-\left|z_{1}-z_{2}\right|^{2}=4 \operatorname{Re} z_{1} \overline{z_{2}},
$$

implies that

$$
\forall \ell \in \mathcal{Q}, \forall k \in \mathbb{R}_{+}, \forall L \geq 0, \delta_{L}(\ell, k)<1 .
$$

Then the convergence follows from Lebesgue's and Parseval's theorems.

Remark 2.2. The study extends to Neumann boundary conditions replacing (2.4) with cosine series. 
2.3. Notations. In computations, the frequency interval $K=\left[k_{\min }, k_{\max }\right]$ depends on the geometry of the domain and the size of the discretization. In each direction $y_{j}$, let $\Delta y_{j}$ denote the length of the mesh. Then $\left(q_{j}\right)_{\min }=1$ and $\left(q_{j}\right)_{\max }=\frac{b_{j}-a_{j}}{\Delta y_{j}}$. Then

$$
k_{\min }=\pi \sqrt{\sum \frac{1}{\left(b_{j}-a_{j}\right)^{2}}}, \quad k_{\max }=\pi \sqrt{\sum \frac{1}{\Delta y_{j}^{2}}} .
$$

In the analysis, we will also consider the case where $k_{\max }=+\infty$, which is relevant in the overlapping case only.

Notation 2.3. We adopt a geometric point of view. When $k$ runs through $K, \omega(k)$ runs through a branch curve $\Gamma$ which relates $\omega_{\min }=\omega\left(k_{\min }\right)$ to $\omega_{\max }=\omega\left(k_{\max }\right)$, see Figure 2.1. $\Gamma$ is a branch of the hyperbola $x y=\mu$ included in the cone $\mathscr{A}$ defined by

$$
\begin{gathered}
\left.\theta_{\min }=\operatorname{Arg}\left(\omega_{\min }\right) \in\right] 0, \frac{\pi}{2}\left[, \quad \theta_{\max }=\operatorname{Arg}\left(\omega_{\max }\right)<\theta_{\min },\right. \\
\mathscr{A}=\left\{z \in \mathbb{C}, \operatorname{Arg} z \in\left[\theta_{\max }, \theta_{\min }\right]\right\} .
\end{gathered}
$$

The focal axis of the hyperbola is $\left\{\theta=\frac{\pi}{4}\right\}$ (black dashed on Figure 2.1). A point on $\Gamma$ is $\omega=x+i y$, its argument is $\theta$, with $\tan \theta=\frac{y}{x}=\frac{\mu}{x^{2}}$. The Robin parameter is $\ell=\ell_{x}+i \ell_{y}$, its argument is $\phi$, and $\tau=\tan \phi=\frac{\ell_{y}}{\ell_{x}}$.

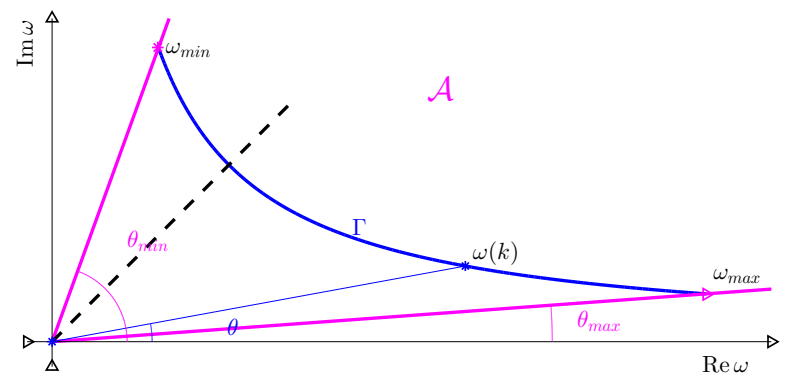

Fig. 2.1: Definition of $\Gamma=\{\omega(k), k \in K\}$.

Since the segment $K$ and the curve $\Gamma$ are in bijection, we shall use indifferently the notation $\delta_{L}(\ell, k)$ for $k \in K$ or $\delta_{L}(\ell, \omega)$ for $\omega \in \Gamma$ when no confusion can be feared, as for instance

$$
h_{L}(\ell)=\sup _{k \in K} \delta_{L}(\ell, k)=\sup _{\omega \in \Gamma} \delta_{L}(\ell, \omega), \quad \delta_{L}^{*}=\inf _{\ell \in \mathbb{C}} \sup _{\omega \in \Gamma} \delta_{L}(\ell, \omega)=\inf _{\ell \in \mathbb{C}} h_{L}(\ell) .
$$

Following [7], it is also useful to introduce the sets

$$
\mathcal{C}(\delta)=\left\{z \in \mathbb{C},\left|\frac{z-1}{z+1}\right|=\delta\right\} \quad \text { and } \quad \mathcal{D}(\delta)=\left\{z \in \mathbb{C},\left|\frac{z-1}{z+1}\right|<\delta\right\},
$$

since, for any $\omega$ and $\ell$,

$$
\delta_{0}(\ell, \omega) \leq \delta \Longleftrightarrow \frac{\ell}{\omega} \in \mathcal{D}(\delta)
$$

The open disk of center $z_{0}$ and radius $r$ is denoted by $B\left(z_{0}, r\right)$, the closed disk is 

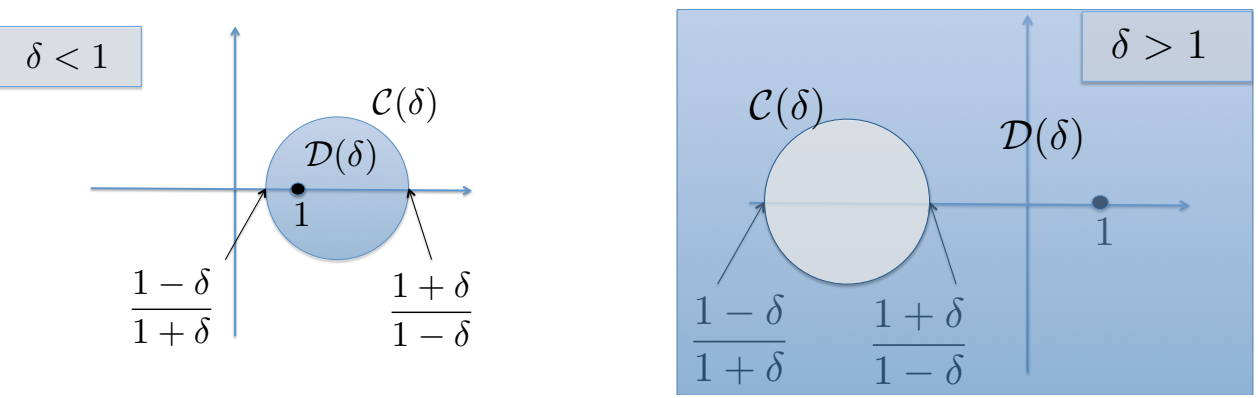

Fig. 2.2: Definition of $\mathcal{D}(\delta)$ in blue grey.

$\bar{B}\left(z_{0}, r\right)$ and the circle is $C\left(z_{0}, r\right)$. If $\delta=1$, the set $\mathcal{C}(\delta)$ is the imaginary line and the set $\mathcal{D}(\delta)$ is the half plane

$$
\Pi_{+}=\{z \in \mathbb{C}, \operatorname{Re}(z)>0\} .
$$

If $\delta \neq 1$, the set $\mathcal{C}(\delta)$ is the circle of center $z_{\delta}=\frac{1+\delta^{2}}{1-\delta^{2}}$ and of radius $r_{\delta}=\frac{2 \delta}{\left|\delta^{2}-1\right|}$. If $0<\delta<1, \mathcal{D}(\delta)$ is the interior of $\mathcal{C}(\delta)$ (namely the disk of radius $r_{\delta}$ and center $z_{\delta}$ ), whereas for $\delta>1$ it is the exterior of this disk. In the latter case, $z_{\delta}+r_{\delta}<0$, which implies that the set $\mathcal{D}(\delta)$ contains $\Pi_{+}$. See Figure 2.2.

We first show that the infimum in $\ell$ over $\mathbb{C}$ defining $\delta_{L}^{*}$ in (2.9) is to be searched in $\mathscr{A}$ only.

THEOREM 2.4 .

$$
\forall L \geq 0, \quad \delta_{L}^{*}:=\inf _{\ell \in \mathbb{C}} \sup _{\omega \in \Gamma} \delta_{L}(\ell, \omega)=\inf _{\ell \in \mathscr{A}} \sup _{\omega \in \Gamma} \delta_{L}(\ell, \omega) .
$$

Furthermore the minimum is reached in the interior $\mathscr{A}$ of $\mathscr{A}$. For $k_{\max } \in \mathbb{R}, \delta_{0}^{*}<1$, and for $k_{\max } \in \overline{\mathbb{R}}, \delta_{L}^{*} \leq e^{-L \omega_{\min }}$.

Proof. A little computation gives the useful formula: for any $\omega \in \mathbb{C}$, for any $\left(\ell_{1}, \ell_{2}\right) \in \mathbb{C}$ such that $\left|\ell_{1}\right|=\left|\ell_{2}\right|, \ell_{1} \neq-\omega$, and $\ell_{2} \neq-\omega$,

$$
\delta_{L}\left(\ell_{1}, \omega\right)^{2}-\delta_{L}\left(\ell_{2}, \omega\right)^{2}=\frac{4\left(|\omega|^{2}+\left|\ell_{1}\right|^{2}\right)}{\left|\ell_{1}+\omega\right|^{2}\left|\ell_{2}+\omega\right|^{2}} \operatorname{Re}\left(\left(\ell_{2}-\ell_{1}\right) \bar{\omega}\right) e^{-L \operatorname{Re} \omega}
$$

Furthermore, formula (2.7) can be rewritten as

$$
\forall \ell \in \mathcal{Q}, \forall \omega \in \Gamma, \delta_{0}(\ell, \omega)=\left|\frac{\omega-\ell}{\omega+\ell}\right|<1 .
$$

The first step is to show that the infimum in $\ell$ can be reduced to $\overline{\mathcal{Q}}$. Choose $\ell \in \mathcal{Q}$ and $\omega \in \Gamma$.

1. For $\ell_{1}=\bar{\ell}$ (symmetry with respect to the real axis),

$$
\ell_{1}-\ell=-2 i \operatorname{Im} \ell, \quad \operatorname{Re}\left(\left(\ell_{1}-\ell\right) \bar{\omega}\right)=-2 \operatorname{Im} \ell \operatorname{Im} \omega<0 .
$$

2. For $\ell_{2}=-\bar{\ell}$ (symmetry with respect to the imaginary axis),

$$
\ell_{2}-\ell=-2 \operatorname{Re} \ell, \quad \operatorname{Re}\left(\left(\ell_{2}-\ell\right) \bar{\omega}\right)=-2 \operatorname{Re} \ell \operatorname{Re} \omega<0 .
$$


3. For $\ell_{3}=-\ell$ (symmetry with respect to the origin),

$$
\ell_{3}-\ell=-2 \ell, \quad \operatorname{Re}\left(\left(\ell_{3}-\ell\right) \bar{\omega}\right)=-2 \operatorname{Re}(\ell \bar{\omega})<0 .
$$

Apply now (2.14) successively to $\left(\ell, \ell_{1}\right),\left(\ell, \ell_{2}\right)$ and $\left(\ell, \ell_{3}\right)$ to obtain that,

$$
\forall \ell \in \mathcal{Q}, \forall \omega \in \Gamma, \quad \delta_{L}(\ell, \omega)<\delta_{L}\left(\ell_{j}, \omega\right), \quad j=1,2,3 .
$$

Taking the supremum on $\Gamma$ gives

for $j=1,2,3, \forall \ell \in \Gamma, \quad h_{L}(\ell) \leq h_{L}\left(\ell_{j}\right), \quad h_{L}(\ell)<h_{L}\left(\ell_{j}\right)$ if $\Gamma$ is compact.

Since the symmetries are involutive, this implies that for any $\tilde{\ell} \notin \mathcal{Q}$, there exists an $\ell$ in $\overline{\mathcal{Q}}$ obtained by one of the reflections above, such that $h_{L}(\tilde{\ell}) \geq h_{L}(\ell)$, proving that

$$
\inf _{\ell \in \mathbb{C}} h_{L}(\ell)=\inf _{\ell \in \overline{\mathcal{Q}}} h_{L}(\ell) .
$$

Reduce now the minimisation domain to $\mathscr{A}$. For any $\ell=|\ell| e^{i \phi} \in \mathcal{Q} \backslash \mathscr{A}$ with $\phi>\theta_{\min }$, let $\ell^{\prime}=|\ell| e^{i \theta_{\min }}$. Then, for any $\omega=|\omega| e^{i \theta} \in \Gamma$, since the cosine function is decreasing on $\left[0, \frac{\pi}{2}\right]$,

$$
\operatorname{Re}\left(\ell-\ell^{\prime}\right) \bar{\omega}=|\ell||\omega|\left(\cos (\phi-\theta)-\cos \left(\theta_{\min }-\theta\right)\right)<0,
$$

which implies by $(2.14)$ that $\delta_{L}\left(\ell^{\prime}, \omega\right)<\delta_{L}(\ell, \omega)$, and therefore $h_{L}\left(\ell^{\prime}\right) \leq h_{L}(\ell)$.

A similar computation holds for $\phi<\theta_{\max }$ (in the case where $\theta_{\max }>0$ ), and the previous steps all together prove (2.13).

Furthermore, let $\ell \in \mathscr{A}$ with $\phi=\theta_{\min }-\varepsilon$. It is easy to see that for any $\omega \in \Gamma$,

$$
\operatorname{Re}\left(\ell-|\ell| e^{i \theta_{\min }}\right) \bar{\omega} \sim|\ell||\omega| \varepsilon \sin \left(\theta_{\min }-\theta\right)>0,
$$

and therefore the minimum of $h_{L}$ is reached in the interior of $\mathscr{A}$. Now use (2.15). If $k_{\max } \in \mathbb{R}_{+}, \Gamma$ is compact, the upperbound $h_{0}(\ell)$ of $\omega \mapsto \delta_{0}(\ell, \omega)$ over $\Gamma$ is smaller than 1 , and therefore $\delta_{0}^{*}=\inf h_{0}(\ell)<1$. In the overlapping case $L>0$, if $k_{\max } \in \overline{\mathbb{R}}_{+}$, for $\ell \in \mathscr{A}$, since $\Gamma$ is included in $\mathscr{A}$,

$$
\delta_{L}(\ell, \omega)=\delta_{0}(\ell, \omega) e^{-L \operatorname{Re} \omega}, \quad \sup _{\omega \in \Gamma} \delta_{L}(\ell, \omega) \leq e^{-L \operatorname{Re} \omega_{\min }} \sup _{\omega \in \Gamma} \delta_{0}(\ell, \omega) \leq e^{-L \operatorname{Re} \omega_{\min }},
$$

and therefore $\delta_{L}^{*}=\inf h_{L}(\ell) \leq e^{-L \operatorname{Re} \omega_{\min }}$.

\subsection{The non-overlapping case $L=0$.}

THEOREM 2.5. Suppose $k_{\max }<+\infty$ and $L=0$. Then there exists a unique $\ell_{0}^{*} \in \mathscr{A}$ such that

$$
\delta_{0}^{*}=\sup _{\omega \in \Gamma} \delta_{0}\left(\ell_{0}^{*}, \omega\right)<1 .
$$

Moreover there exists at least two points $\omega_{1}^{*}$ and $\omega_{2}^{*}$ on $\Gamma$ such that

$$
\delta_{0}^{*}=\delta_{0}\left(\ell_{0}^{*}, \omega_{1}^{*}\right)=\delta_{0}\left(\ell_{0}^{*}, \omega_{1}^{*}\right) .
$$

Remark 2.6. If $k_{\max }=+\infty$, the upperbound over $K$ in (1.3) is 1 for any $\ell$. Therefore the convergence factor is 1 , and the optimization problem makes sense only if $k_{\max }<+\infty$.

Proof. Existence, equioscillation property, and uniqueness are contained in gen-

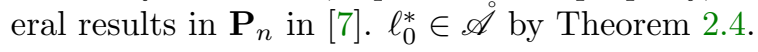

TheOrem 2.7. Assume that $k_{\max }<+\infty$ and $L=0$. Then the function $h_{0}$ in (2.9) is continuous, and any strict local minimum for $h_{0}$ is the global minimum.

The function $h_{0}$ is continuous since $\Gamma$ is a compact set in $\mathbb{C}$. The remainder of the statement is verbatim in [7, Theorem 2.7]. 
2.5. The Overlapping case $L \neq 0$. In reference [7], best approximation results in $\mathbb{P}_{n}$ for a positive overlap $L$ were proved with a very strong restriction on the size of the overlap. Here we present in the case $n=0$ the first proof of a general result valid for any size of the overlap, and for unbounded intervals $K$.

Theorem 2.8. For any $L>0$, for $k_{\max } \in \overline{\mathbb{R}}$,

$$
\exists A>0, B>0 \text { such that } \delta_{L}^{*}=\inf _{\substack{\ell \in A \\|\ell| \leq A}} \sup _{\substack{\omega \in \Gamma \\|\omega| \leq B}} \delta_{L}(\ell, \omega) .
$$

The function $h_{L}$ in (2.9) is continuous, and there exists a unique $\ell_{L}^{*} \in \mathscr{A}$ such that

$$
\delta_{L}^{*}=\sup _{\omega \in \Gamma} \delta_{L}\left(\ell_{L}^{*}, \omega\right) .
$$

Furthermore there exists at least two distinct points $\omega_{1}^{*}$ and $\omega_{2}^{*}$ on $\Gamma$ such that

$$
\delta_{L}^{*}=\delta_{L}\left(\ell_{L}^{*}, \omega_{1}^{*}\right)=\delta_{L}\left(\ell_{L}^{*}, \omega_{2}^{*}\right) .
$$

Proof. Existence. By Theorem 2.4, the infimum has to be found in $\mathscr{A}$, and for $\ell \in \mathscr{A}$, for any $\omega \in \Gamma, \delta_{0}(\ell, \omega) \leq 1$ and $\delta_{L}(\ell, \omega) \leq e^{-L \operatorname{Re} \omega_{\min }}<1$. We now reduce the min-max problem on unbounded sets to a min-max problem on compact sets. It will be done in two steps:

1. We will show that the sup in (1.3) is reached on a compact set uniformly in $\ell$. More precisely, there exists a compact set $\underline{\Gamma} \subset \Gamma$ such that, $\forall \ell \in$ $\mathscr{A}, \sup _{\omega \in \Gamma} \delta(\ell, \omega)=\sup _{\omega \in \Gamma} \delta(\ell, \omega)$.

2. Supposing now $\Gamma$ bounded, we will reduce the minimization of $h_{L}$ to a compact subset of $\mathscr{A}$.

1. Fix $\ell \in \mathscr{A}$. Since $\omega \mapsto \delta_{L}(\ell, \omega)$ is smaller than 1 and tends to zero as $\omega$ tends to infinity on $\Gamma$, the function has a maximum on $\Gamma$ reached for $\tilde{\omega}(\ell)$. Then

$$
h_{L}(\ell)=\delta_{L}(\ell, \tilde{\omega}(\ell))=\delta_{0}(\ell, \tilde{\omega}(\ell)) e^{-L \operatorname{Re} \tilde{\omega}(\ell)} .
$$

If $\ell \neq \omega_{\min }$, write that $h_{L}(\ell) \geq \delta_{L}\left(\ell, \omega_{\min }\right)$ :

$$
\left|\delta_{0}(\ell, \tilde{\omega}(\ell))\right| e^{-L \operatorname{Re} \tilde{\omega}(\ell)} \geq\left|\delta_{0}\left(\ell, \omega_{\min }\right)\right| e^{-L \operatorname{Re} \omega_{\min }} .
$$

Rewrite the previous inequality as

$$
e^{L\left(\operatorname{Re} \tilde{\omega}(\ell)-\operatorname{Re} \omega_{\min }\right)} \leq \frac{\left|\delta_{0}(\ell, \tilde{\omega}(\ell))\right|}{\left|\delta_{0}\left(\ell, \omega_{\min }\right)\right|}
$$

and take the logarithm,

$$
L\left(\operatorname{Re} \tilde{\omega}(\ell)-\operatorname{Re} \omega_{\min }\right) \leq \ln \left|\delta_{0}(\ell, \tilde{\omega}(\ell))\right|-\ln \left|\delta_{0}\left(\ell, \omega_{\min }\right)\right| .
$$

Since $\left|\delta_{0}(\ell, \tilde{\omega}(\ell))\right|<1$, we deduce that

$$
L\left(\operatorname{Re} \tilde{\omega}(\ell)-\operatorname{Re} \omega_{\min }\right) \leq-\ln \left|\delta_{0}\left(\ell, \omega_{\min }\right)\right| .
$$

Furthermore since $\tilde{\omega}(\ell) \in\left[\omega_{\min }, \omega_{\max }\right], \operatorname{Re} \tilde{\omega}(\ell)$ is larger than $\operatorname{Re} \omega_{\min }$, see Figure 2.1. These two observations lead to the key inequality

$$
\operatorname{Re} \omega_{\min } \leq \operatorname{Re} \tilde{\omega}(\ell) \leq \operatorname{Re} \omega_{\min }+\frac{1}{L} \ln \left|\frac{\ell+\omega_{\min }}{\ell-\omega_{\min }}\right| .
$$


If $\ell$ becomes large, the term on the far right is small, and for instance

$$
\exists r_{0}>0, \forall \ell \in \mathscr{A},|\ell|>r_{0} \Longrightarrow \ln \left|\frac{\ell+\omega_{\min }}{\ell-\omega_{\min }}\right|<L \operatorname{Re} \omega_{\min } .
$$

Using the right inequality in $(2.22)$, choosing $r_{1}=\max \left(r_{0},\left|\omega_{\min }\right|\right)$, this proves that

$$
\exists r_{1}>0, \forall \ell \in \mathscr{A},|\ell|>r_{1} \Longrightarrow \operatorname{Re} \tilde{\omega}(\ell)<C_{1}=2 \operatorname{Re} \omega_{\min } .
$$

$\tilde{\omega}$ is a continuous function of $\ell$, it is bounded over the compact set $\mathscr{A} \cap\{|\ell| \leq$ $\left.r_{1}\right\}$.

$$
\exists C_{2}>0, \forall \ell \in \mathscr{A} \cap\left\{|\ell| \leq r_{1}\right\}, \quad \operatorname{Re} \tilde{\omega}(\ell)<C_{2} .
$$

Therefore $\operatorname{Re} \tilde{\omega}(\ell)$ is bounded over $\mathscr{A}$ by $B=\max \left(C_{1}, C_{2}\right)$, and defining $\underline{\Gamma}=\Gamma \cap\{|\omega| \leq B\}$,

$$
\forall \ell \in \mathscr{A}, \quad \sup _{\omega \in \Gamma} \delta_{L}(\ell, \omega)=\sup _{\omega \in \underline{\Gamma}} \delta_{L}(\ell, \omega),
$$

which implies

$$
\inf _{\ell \in \mathscr{A}} \sup _{\omega \in \Gamma} \delta_{L}(\ell, \omega)=\inf _{\ell \in \mathscr{A}} \sup _{\omega \in \underline{\Gamma}} \delta_{L}(\ell, \omega) .
$$

2. We now show that the minimization in $\ell$ can be reduced to a bounded set. As stated in Theorem 2.4, an upper bound for $\delta_{L}(\ell, \omega)$ is $e^{-L \operatorname{Re} \omega_{\min }}$. Choose $\ell_{1} \in \mathscr{A}$, and $\tilde{\omega}\left(\ell_{1}\right)$ as in $(2.21)$. There exists $\varepsilon>0$ such that $\delta_{0}\left(\ell_{1}, \tilde{\omega}\left(\ell_{1}\right)\right)<$ $1-\varepsilon$, and hence

$$
\delta_{L}\left(\ell_{1}, \tilde{\omega}\left(\ell_{1}\right)\right)<(1-\varepsilon) e^{-L \operatorname{Re} \tilde{\omega}\left(\ell_{1}\right)}<(1-\varepsilon) e^{-L \operatorname{Re} \omega_{\min }} .
$$

Besides, as $\ell$ tends to infinity, $\delta_{0}(\ell, \tilde{\omega}(\ell))$ tends to 1 , which implies that

$$
\exists C>0,|\ell| \geq C \Longrightarrow(1-\varepsilon / 2) e^{-L \operatorname{Re} \tilde{\omega}(\ell)} \leq \delta_{L}(\ell, \tilde{\omega}(\ell)) \leq e^{-L \operatorname{Re} \tilde{\omega}(\ell)} .
$$

As in the first part, insert into the right inequality of (2.22) the continuity result

$$
\exists C^{\prime}>0, \forall \ell \in \mathscr{A},|\ell|>C^{\prime} \Longrightarrow \ln \left|\frac{\ell+\omega_{\min }}{\ell-\omega_{\min }}\right|<\frac{\varepsilon}{2},
$$

to obtain

$$
\exists C^{\prime}>0, \forall \ell \in \mathscr{A},|\ell| \geq C^{\prime} \Longrightarrow \operatorname{Re} \omega_{\min } \leq \operatorname{Re} \tilde{\omega}(\ell) \leq \operatorname{Re} \omega_{\min }+\frac{\varepsilon}{2 L} .
$$

Plug it in the previous inequality to get that for $|\ell| \geq A=\max \left(C, C^{\prime}\right)$,

$$
(1-\varepsilon / 2) e^{-\varepsilon / 2} e^{-L \operatorname{Re} \omega_{\min }} \leq \delta_{L}(\ell, \tilde{\omega}(\ell)) \leq e^{-L \operatorname{Re} \omega_{\min }},
$$

which implies

$$
(1-\varepsilon) e^{-L \operatorname{Re} \omega_{\min }} \leq \delta_{L}(\ell, \tilde{\omega}(\ell)) \leq e^{-L \operatorname{Re} \omega_{\min }} .
$$

Then, plugging the estimate (2.23) in $\ell_{1}$, we obtain

$$
\forall \ell \in \mathscr{A},|\ell| \geq A \Longrightarrow \delta_{L}\left(\ell_{1}, \tilde{\omega}\left(\ell_{1}\right)\right)=h_{L}\left(\ell_{1}\right)<\delta_{L}(\ell, \tilde{\omega}(\ell))=h_{L}(\ell) .
$$

Therefore the infimum of $h_{L}$ over $\mathscr{A}$ is the infimum over the compact set $\mathscr{A} \cap\{|\ell| \leq A\}$. 
The key compactness result (2.18) is proved. Since $\underline{\Gamma}=\{\omega \in \Gamma,|\omega| \leq B\}$ is compact, the function $h_{L}$ is continuous, therefore reaches its minimum over the compact set $\mathscr{A} \cap\{|\ell| \leq A\}$. This gives existence. By compactness again, there exists $\omega_{1}^{*}$ such that

$$
\delta_{L}^{*}=\delta_{L}\left(\ell_{L}^{*}, \omega_{1}^{*}\right)
$$

Equioscillation. The proof is rather long but is verbatim the proof in $[7$, Theorem 2.11].

Uniqueness. The proof relies on convexity as in the Chebyshev theory: we first show that the set of best approximations is convex, and then prove the uniqueness by contradiction. It is an extension of the proof of Theorem 8 in [6].

Since $\Gamma \subset \mathscr{A}, \ell_{L}^{*}$ is an optimal solution if and only if

$$
\ell_{L}^{*} \in \mathscr{A} \text { and } \sup _{\omega \in \Gamma}\left(\delta_{0}\left(\ell_{L}^{*}, \omega\right) e^{-L \operatorname{Re} \omega}\right)=\delta_{L}^{*},
$$

in other words

$$
\ell_{L}^{*} \in \mathscr{A} \text { and } \forall \omega \in \Gamma, \frac{\ell^{*}}{\omega} \in \mathcal{D}\left(e^{L \operatorname{Re} \omega} \delta_{L}^{*}\right) .
$$

Furthermore it has already been noticed that for any $\omega \in \Gamma$, Re $\ell_{L}^{*} \bar{\omega}>0$. Define a function $\rho$ on $\Gamma$ by

$$
\begin{gathered}
\forall \omega \in \Gamma, \quad \rho(\omega):=e^{L \operatorname{Re} \omega} \delta_{L}^{*} . \\
\ell^{*} \text { optimal solution } \Longrightarrow \forall \omega \in \Gamma, \frac{\ell^{*}}{\omega} \in \mathcal{D}_{\omega}^{+}:=\Pi_{+} \cap \mathcal{D}(\rho(\omega)) .
\end{gathered}
$$

$\Pi_{+}$is the half-plane defined in (2.12). For any $\omega, \mathcal{D}_{\omega}^{+}$is convex, see Figure 2.2: if $\rho(\omega)<1, \mathcal{D}(\rho(\omega)) \subset \Pi_{+}$, hence $\mathcal{D}_{\omega}^{+}=\mathcal{D}(\rho(\omega))$ is convex. If $\rho(\omega) \geq 1, \mathcal{D}(\rho(\omega)) \supset \Pi_{+}$, hence $\mathcal{D}_{\omega}^{+}=\Pi_{+}$is convex.

The set of best approximations is convex. Let $\ell^{*}$ and $\tilde{\ell}^{*}$ be two optimal parameters, let us show that any $\ell$ in the segment $\left[\ell^{*}, \tilde{\ell}^{*}\right]$ is optimal as well. By property (2.24), for all $\omega \in \Gamma, \frac{\ell^{*}}{\omega}$ and $\frac{\tilde{\ell}^{*}}{\omega}$ both belong to $\mathcal{D}_{\omega}^{+}$which is convex. Therefore $\frac{\ell}{\omega}$ is in $\mathcal{D}_{\omega}^{+}$, and satisfies

$$
h_{L}(\ell)=\sup _{\omega \in \Gamma} \delta_{L}(\ell, \omega) \leq \delta_{L}^{*} .
$$

Since $\delta_{L}^{*}$ is the minimum of $h_{L}$, this implies equality, and $\ell$ is an optimal parameter.

Uniqueness of the best parameter. Assume again that $\ell^{*}$ and $\tilde{\ell}^{*}$ are two optimal parameters and, for any $\theta \in] 0,1\left[\right.$, define $\ell_{\theta}=\theta \ell^{*}+(1-\theta) \tilde{\ell}^{*}$ which is also a best parameter. Applying the equioscillation property to $\ell_{\theta}$, there exist $\omega_{1}$ and $\omega_{2}$ on $\Gamma$ such that

$$
\left|\frac{\omega_{i}-\ell_{\theta}}{\omega_{i}+\ell_{\theta}}\right|=\rho\left(\omega_{i}\right), i=1,2
$$

Therefore for $i=1,2$,

$$
\frac{\ell^{*}}{\omega_{i}} \in \mathcal{D}\left(\rho\left(\omega_{i}\right)\right) \cap \Pi_{+}, \quad \frac{\tilde{\ell}^{*}}{\omega_{i}} \in \mathcal{D}\left(\rho\left(\omega_{i}\right)\right) \cap \Pi_{+}, \quad \frac{\ell_{\theta}}{\omega_{i}} \in \mathcal{C}\left(\rho\left(\omega_{i}\right)\right) \cap \Pi_{+} .
$$

If for $i=1$ or $i=2, \rho\left(\omega_{i}\right)>1$, then $\mathcal{C}\left(\rho\left(\omega_{i}\right)\right) \subset \Pi_{-}$, therefore $\mathcal{C}\left(\rho\left(\omega_{i}\right)\right) \cap \Pi_{+}=\emptyset$, which is impossible. Then for $i=1,2, \rho\left(\omega_{i}\right) \leq 1$, and therefore $\mathcal{D}\left(\rho\left(\omega_{i}\right)\right) \cap \Pi_{+}=\mathcal{D}\left(\rho\left(\omega_{i}\right)\right)$. 
If $\rho\left(\omega_{i}\right)=1$, then $\mathcal{D}\left(\rho\left(\omega_{i}\right)\right) \cap \Pi_{+}=\Pi_{+}$and $\mathcal{C}\left(\rho\left(\omega_{i}\right)\right) \cap \Pi_{+}=i \mathbb{R}$. Then (2.25) implies that $\frac{\tilde{\ell}^{*}}{\omega_{i}}$ and $\frac{\ell^{*}}{\omega_{i}}$ are also in $i \mathbb{R}$. This again is impossible since their real parts are positive.

If $\rho\left(\omega_{i}\right)<1$ for $i=1,2, \frac{\ell_{\theta}}{\omega_{i}}$ is on the circle $\mathcal{C}\left(\rho\left(\omega_{i}\right)\right) \subset \Pi_{+}$, while $\frac{\tilde{\ell}^{*}}{\omega_{i}}$ and $\frac{\ell^{*}}{\omega_{i}}$ are in the disc. Therefore they coincide

$$
\frac{\ell^{*}}{\omega_{i}}=\frac{\tilde{\ell}^{*}}{\omega_{i}}=\frac{\ell_{\theta}}{\omega_{i}}, \quad i=1,2,
$$

which proves that $\ell^{*}=\tilde{\ell}^{*}$.

Theorem 2.9. Any strict local minimum of $h_{L}$ in $\mathscr{A}$ is the unique global minimum of $h_{L}$.

Proof. The same technique as in the previous theorem shows that for positive $\delta<1$, the set $\mathcal{D}_{\delta}=\left\{\ell \in \mathscr{A}, h_{L}(\ell)<\delta\right\}$ is convex. Suppose now that $\left(\hat{\ell}, \hat{\delta}=h_{L}(\hat{\ell})\right)$ is a strict local minimum of $h_{L}$, but not the global minimum, that is $\delta_{L}^{*}<\hat{\delta}$. Then $\ell_{L}^{*}$ and $\hat{\ell}$ are both in $\mathcal{D}_{\hat{\delta}}$, therefore the segment $\left[\ell_{L}^{*}, \hat{\ell}\right]$ is in $\mathcal{D}_{\hat{\delta}}$ : for all $\theta \in[0,1]$, $\ell_{\theta}=\hat{\ell}+\theta\left(\ell_{L}^{*}-\hat{\ell}\right) \in \mathcal{A}$, and $h_{L}\left(\ell_{\theta}\right) \leq \hat{\delta}$.

But since $\hat{\ell}$ is a strict local minimum, for sufficiently small $\theta, h_{L}\left(\ell_{\theta}\right)>\hat{\delta}$, and a contradiction is reached.

3. Characterization of the optimal parameter. In order to compute the optimal parameters, we need to identify the equioscillation points, which are amongst the local extrema of $\delta_{L}$ in the $k$ variable. For this part of the analysis, it is more convenient to use the convergence factor

$$
\mathscr{R}_{L}(\ell, \omega)=\delta_{L}(\ell, \omega)^{2} .
$$

From $\omega=x+i y$ on the curve $\Gamma, x$ is a strictly increasing function of $k$, and $x y=\mu$. Then we can rewrite $\mathscr{R}_{L}$ as a function of the increasing variable $x$ only,

$$
\mathscr{R}_{L}(\ell, x)=\frac{x^{2}\left(x-\ell_{x}\right)^{2}+\left(\mu-x \ell_{y}\right)^{2}}{x^{2}\left(x+\ell_{x}\right)^{2}+\left(\mu+x \ell_{y}\right)^{2}} e^{-2 L x}, \ell=\ell_{x}+i \ell_{y}, \tau=\frac{\ell_{y}}{\ell_{x}} .
$$

As for $\delta_{L}$, we will write according to the circumstances $\mathscr{R}_{L}(\ell, x)$ or $\mathscr{R}_{L}(\ell, \omega)$.

3.1. Variation of $\mathscr{R}_{L}$ with respect to $x$ and $\ell$. The derivative of $\mathscr{R}_{L}$ with respect to $x$ is given by

$$
\frac{\partial}{\partial x} \mathscr{R}_{L}(\ell, x)=\frac{4 q_{L}\left(\ell, x^{2}\right)}{\left(x^{2}\left(x+\ell_{x}\right)^{2}+\left(\mu+x \ell_{y}\right)^{2}\right)^{2}} e^{-2 L x} .
$$

The numerator $q_{L}$ is a polynomial of degree 4 , for $L>0$, of degree 3 for $L=0$. Its roots define the local extrema of $x \mapsto \mathscr{R}_{L}(\ell, x)$. It is given by

Notation 3.1.

$$
\omega=x+i y \in \Gamma, t=x^{2}, \theta=\arg \omega, \tan \theta=\frac{\mu}{t}, \ell=\ell_{x}+i \ell_{y},
$$

$$
\begin{aligned}
& q_{L}(\ell, t)=q_{0}(\ell, t)+L \tilde{q}(\ell, t) \\
& q_{0}(\ell, t)=\ell_{x} t^{3}+\left(3 \mu \ell_{y}-\ell_{x}|\ell|^{2}\right) t^{2}+\mu\left(|\ell|^{2} \ell_{y}-3 \mu \ell_{x}\right) t-\mu^{3} \ell_{y} \\
& \tilde{q}(\ell, t)=-\frac{1}{2} t^{4}+\left(\ell_{x}^{2}-\ell_{y}^{2}\right) t^{3}-\frac{1}{2}\left(2 \mu^{2}-8 \mu \ell_{x} \ell_{y}+\left(\ell_{x}^{2}+\ell_{y}^{2}\right)^{2}\right) t^{2}-\mu^{2}\left(\ell_{x}^{2}-\ell_{y}^{2}\right) t-\frac{1}{2} \mu^{4} .
\end{aligned}
$$


The second order Taylor-Young expansion of $\mathscr{R}_{L}$ in $\ell$ will be useful in the analysis. It is obtained most easily by expanding directly $\mathscr{R}_{L}(\ell, \omega)$, as

$$
\frac{\mathscr{R}_{L}(\ell+\varepsilon \xi, \omega)}{\mathscr{R}_{L}(\ell, \omega)}=1+4 \varepsilon \operatorname{Re}(\xi V(\ell, \omega))+4 \varepsilon^{2} R_{2}(\ell, \omega, \xi)+\mathcal{O}\left(\varepsilon^{3}\right) \xi^{3} .
$$

with

$$
V(\ell, \omega)=\frac{\omega}{\ell^{2}-\omega^{2}}, \quad R_{2}(\ell, \omega, \xi)=|\xi V(\ell, \omega)|^{2}-\operatorname{Re} \frac{\xi^{2} V(\ell, \omega)}{\ell+\omega} .
$$

The formula for $R_{2}$ can be simplified into a quadratic form in $\xi V(\ell, \omega)$ :

$$
R_{2}(\ell, \omega, \xi)=|\xi V(\ell, \omega)|^{2}-\operatorname{Re}\left((\xi V(\ell, \omega))^{2} \frac{\ell-\omega}{\omega}\right):=Q(\ell, \omega, \xi V(\ell, \omega)) .
$$

Using the canonical isomorphism between $\mathbb{R}^{2}$ and $\mathbb{C}$, the derivative in $\mathbb{R}^{2}$ with respect to $\left(\ell_{x}, \ell_{y}\right)$ called $D_{\ell} \mathscr{R}_{L}(\ell, \omega)$ can also be identified from the expansion, and all this is summarized below:

$$
\begin{aligned}
& V(\ell, \omega)=\frac{\omega}{\ell^{2}-\omega^{2}}, \quad D_{\ell} \mathscr{R}_{L}(\ell, \omega)=4 \mathscr{R}_{L}(\ell, \omega) \overline{V(\ell, \omega)}, \\
& Q(\ell, \omega, Z)=|Z|^{2}-\operatorname{Re}\left(\frac{\ell-\omega}{\omega} Z^{2}\right), \\
& \frac{\mathscr{R}_{L}(\ell+\varepsilon \xi, \omega)}{\mathscr{R}_{L}(\ell, \omega)}=1+4 \varepsilon \operatorname{Re}(\xi V(\ell, \omega))+4 \varepsilon^{2} Q(\ell, \omega, \xi V(\ell, \omega))+\mathcal{O}\left(\varepsilon^{3}\right) \xi^{3} .
\end{aligned}
$$

\subsection{Alternation: a sufficient condition for optimum.}

Theorem And Definition 3.2. For any $L \geq 0$, let $\hat{\ell} \in \mathscr{A}$ such that there are two alternation points $\omega_{j}$ on $\Gamma$, that is such that the two properties below are fulfilled,

$$
\begin{gathered}
\delta_{L}\left(\hat{\ell}, \omega_{1}\right)=\delta_{L}\left(\hat{\ell}, \omega_{2}\right)=\sup _{\omega \in \Gamma} \mathscr{R}_{L}(\hat{\ell}, \omega), \\
\exists p \in \mathbb{R}_{+}^{*}, V\left(\hat{\ell}, \omega_{2}\right)+p V\left(\hat{\ell}, \omega_{1}\right)=0 .
\end{gathered}
$$

Then $\hat{\ell}$ is a local strict minimum point for the function $h_{L}$ defined in (2.9).

Proof. Using the function $\mathscr{R}_{L}, \hat{\ell}$ is a local strict minimum point for $h_{L}$ if there exists $\varepsilon>0$ such that, for any $\xi \in \mathbb{C}$ with $|\xi| \leq 1, \xi \neq 0$,

$$
\sup _{\omega \in \Gamma} \mathscr{R}_{L}(\hat{\ell}+\varepsilon \xi, \omega)>\sup _{\omega \in \Gamma} \mathscr{R}_{L}(\hat{\ell}, \omega)=\mathscr{R}_{L}\left(\hat{\ell}, \omega_{1}\right)=\mathscr{R}_{L}\left(\hat{\ell}, \omega_{2}\right) .
$$

By continuity, it is sufficient to prove that

$$
\max \left(\mathscr{R}_{L}\left(\hat{\ell}+\varepsilon \xi, \omega_{1}\right), \mathscr{R}_{L}\left(\hat{\ell}+\varepsilon \xi, \omega_{2}\right)\right)>\mathscr{R}_{L}\left(\hat{\ell}, \omega_{1}\right)=\mathscr{R}_{L}\left(\hat{\ell}, \omega_{2}\right) .
$$

To achieve this result, write the Taylor expansion in (3.4) at points $\omega_{j}$ :

$$
\frac{\mathscr{R}_{L}\left(\hat{\ell}+\varepsilon \xi, \omega_{j}\right)}{\mathscr{R}_{L}\left(\hat{\ell}, \omega_{j}\right)}=1+4 \varepsilon \operatorname{Re}\left(\xi V\left(\hat{\ell}, \omega_{j}\right)\right)+4 \varepsilon^{2} Q\left(\hat{\ell}, \omega_{j}, \xi V\left(\hat{\ell}, \omega_{j}\right)\right)+\mathcal{O}\left(\varepsilon^{3}\right) \xi^{3} .
$$

We need an evaluation of the righthand side. The straight line $D=\overline{V\left(\hat{\ell}, \omega_{1}\right)} \stackrel{\perp}{=}$ $\left\{\xi, \operatorname{Re}\left(\xi V\left(\hat{\ell}, \omega_{1}\right)\right)=0\right\}$ splits the complex plane into two closed half-spaces, see Figure 3.1 ,

$$
\mathcal{D}_{1}=\left\{\xi, \operatorname{Re}\left(\xi V\left(\hat{\ell}, \omega_{1}\right)\right) \geq 0\right\}, \quad \mathcal{D}_{2}=\left\{\xi, \operatorname{Re}\left(\xi V\left(\hat{\ell}, \omega_{2}\right)\right) \geq 0\right\} .
$$


For $\xi \in D$, by the assumption in (3.6), the first order term vanishes in (3.8) for $j=1,2$. Therefore in order to control $\mathscr{R}_{L}\left(\hat{\ell}+\varepsilon \xi, \omega_{j}\right)$ over the full disk $|\xi| \leq 1$, we need to control the second order term as well. Rewrite the quadratic form $Q(\hat{\ell}, \omega, \cdot)$ defined in (3.4) as

$$
\text { for } Z=X+i Y, \quad Q(\hat{\ell}, \omega, Z)=\left(2-\operatorname{Re} \frac{\hat{\ell}}{\omega}\right) X^{2}+2\left(\operatorname{Im} \frac{\hat{\ell}}{\omega}\right) X Y+\left(\operatorname{Re} \frac{\hat{\ell}}{\omega}\right) Y^{2} .
$$

On the axis $D, Z= \pm \xi V\left(\hat{\ell}, \omega_{j}\right) \in i \mathbb{R}$, and $Q\left(\hat{\ell}, \omega_{j}, Z\right)=\left(\operatorname{Re} \frac{\hat{\ell}}{\omega_{j}}\right) Y^{2} \geq 0$ since both $\hat{\ell}$ and $\omega$ are in $\mathscr{A}$. Therefore in each half-plane, the quadratic form is either positive definite (best case), or positive indefinite, vanishing on a line strictly included in the half-plane, or it is hyperbolic, it changes sign on two lines strictly included in the half-plane; this is the worst case, depicted in Figure 3.1. In any case, there is a closed

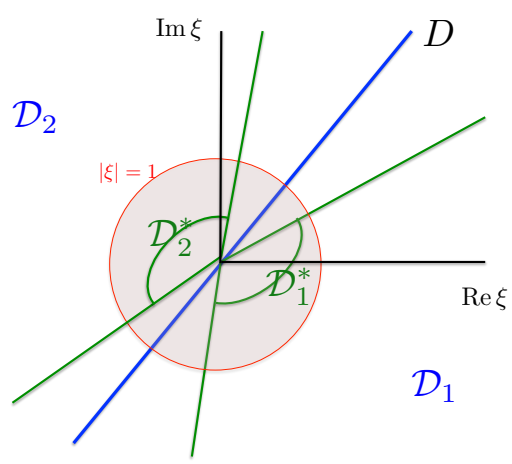

Fig. 3.1: Definition of the domains in the proof of strict local minimum

angular domain $\mathcal{D}_{j}^{*} \subset \mathcal{D}_{j}$ such that the quadratic form is definite positive in $\mathcal{D}_{j} \backslash \mathcal{D}_{j}^{*}$. Consider first $\xi \in C(0,1)$, i.e. $|\xi|=1$. Then

$$
\begin{aligned}
& \exists a_{j}>0, \forall \xi \in \mathcal{D}_{j}^{*}, \operatorname{Re}\left(\xi V\left(\hat{\ell}, \omega_{j}\right)\right) \geq a_{j}, \\
& \exists A_{j}>0, \forall \xi \in \mathcal{D}_{j}^{*}, Q\left(\ell, \omega_{j}, \xi V\left(\hat{\ell}, \omega_{j}\right)\right) \geq-A_{j}, \\
& \exists B_{j}>0, \forall \xi \in \mathcal{D}_{j} \backslash \mathcal{D}_{j}^{*}, Q\left(\hat{\ell}, \omega_{j}, \xi V\left(\hat{\ell}, \omega_{j}\right)\right) \geq B_{j} .
\end{aligned}
$$

which leads to a lower bound of the term in (3.8): for $\xi \in \mathcal{D}_{j} \cap C(0,1)$,

$$
\varepsilon \operatorname{Re}\left(\xi V\left(\hat{\ell}, \omega_{j}\right)\right)+\varepsilon^{2} Q\left(\hat{\ell}, \omega_{j}, \xi V\left(\hat{\ell}, \omega_{j}\right)\right) \geq \begin{cases}\varepsilon a_{j}-\varepsilon^{2} A_{j} & \text { in } \mathcal{D}_{j}^{*}, \\ B_{j} \varepsilon^{2} & \text { in } \overline{\mathcal{D}_{j} \backslash \mathcal{D}_{j}^{*}}\end{cases}
$$

Choose now $\varepsilon \leq \min _{j} \min \left(\frac{1}{2} \frac{a_{j}}{A_{j}}, 1\right)$ to obtain that there exists $C_{j}>0$ such that for $\xi \in \mathcal{D}_{j} \cap C(0,1)$,

$$
\varepsilon \operatorname{Re}\left(\xi V\left(\hat{\ell}, \omega_{j}\right)\right)+\varepsilon^{2} Q\left(\hat{\ell}, \omega_{j}, \xi V\left(\hat{\ell}, \omega_{j}\right)\right) \geq C_{j} \varepsilon^{2} \text { for } j=1,2 .
$$


If now $\xi \in \mathcal{D}_{j} \cap \bar{B}(0,1)$, define $\zeta=\frac{\xi}{\|\xi\|}$, and write

$$
\begin{aligned}
4 \varepsilon \operatorname{Re}\left(\xi V\left(\hat{\ell}, \omega_{j}\right)\right) & +4 \varepsilon^{2} Q\left(\hat{\ell}, \omega_{j}, \xi V\left(\hat{\ell}, \omega_{j}\right)\right) \\
& =4\|\xi\|^{2}\left(\frac{\varepsilon}{\|\xi\|} \operatorname{Re}\left(\zeta V\left(\hat{\ell}, \omega_{j}\right)\right)+\varepsilon^{2} Q\left(\hat{\ell}, \omega_{j}, \zeta V\left(\hat{\ell}, \omega_{j}\right)\right)\right) \\
& \geq 4 C_{j} \varepsilon^{2}\|\xi\|^{2} .
\end{aligned}
$$

Insert into (3.8) to get for $\xi \in \mathcal{D}_{j} \cap \bar{B}(0,1)$,

$$
\frac{\mathscr{R}_{L}\left(\hat{\ell}+\varepsilon \xi, \omega_{j}\right)}{\mathscr{R}_{L}\left(\hat{\ell}, \omega_{j}\right)} \geq 1+4 C_{j} \varepsilon^{2}\|\xi\|^{2}+\mathcal{O}\left(\varepsilon^{3}\right) \xi^{3} .
$$

By compactness, for $\varepsilon$ sufficiently small, and for all $\xi \in \mathcal{D}_{j} \cap \bar{B}(0,1),\left|\mathcal{O}\left(\varepsilon^{3}\right) \xi^{3}\right| \leq 2 C_{j} \varepsilon^{2}\|\xi\|^{2}$ and

$$
\frac{\mathscr{R}_{L}\left(\hat{\ell}+\varepsilon \xi, \omega_{j}\right)}{\mathscr{R}_{L}\left(\hat{\ell}, \omega_{j}\right)} \geq 1+2 C_{j} \varepsilon^{2}\|\xi\|^{2}>1 .
$$

Any $\xi \in \bar{B}(0,1)$ belongs to either set $\mathcal{D}_{j} \cap \bar{B}(0,1)$, and therefore either one of the two lower bounds above is true. Since $\mathscr{R}_{L}\left(\hat{\ell}, \omega_{1}\right)=\mathscr{R}_{L}\left(\hat{\ell}, \omega_{2}\right),(3.7)$ is proved.

3.3. The non-overlapping case $L=0$. By Theorem 2.5, the min-max problem has a unique solution $\ell_{0}^{*}$, the aim of this section is to provide a characterization and an operational formula for this parameter. The local extrema are given by the roots of the third order polynomial $t \mapsto q_{0}(\ell, t)$ defined in (3.3), which has one or three roots. Therefore $x \mapsto \mathscr{R}_{0}(\ell, x)$ has at most one local maximum over $(0,+\infty)$. Proposition 3.5 analyses precisely the roots of $q_{0}$, depending on the position of $\ell$ in $\mathcal{Q}$. When $\ell_{y} \leq \ell_{x}$, we obtain exact results, whereas the general case is analysed asymptotically only.

Remark 3.3. The polynomial $q_{0}$ is of degree 3, and its roots can be written in closed form, using the Cardano formulas [10]. However these formulas are not very tractable in general for a polynomial with coefficients depending on parameters, as it is for instance impossible to compare the roots to decide which one corresponds to a maximum point.

Then based on the equioscillation property in Theorem 2.5, Theorem 3.7 defines a specific equioscillation value $\hat{\ell}$ of the parameter. For $\theta \leq \frac{\pi}{3}, \hat{\ell}$ is given by an explicit formula. For $\theta>\frac{\pi}{3}, \hat{\ell}$ is shown to be solution of a nonlinear equation, and is given asymptotically for large $k_{\max }$. The last step is to use the characterization in Theorem 3.2 to prove that $\hat{\ell}$ is indeed a strict minimum. This is done by a generalized convexity analysis in $\mathbb{C}$.

Notation 3.4. In the vicinity of 0 , we will use for convenience the Landau notation $f=\mathcal{O}(g)$ for comparison: there exists a positive constant $C$ such that for small $h$, $|f(h)| \leq C|g(h)|$. We will say that $f$ and $g$ are of same order, and write $f \approx g$ if there exist two positive constants $C_{1}$ and $C_{2}$ such that for small $h, C_{1} f(h) \leq g(h) \leq C_{2} f(h)$. For positive functions, this is equivalent to $f=\mathcal{O}(g)$ and $g=\mathcal{O}(h)$. We will say that $f$ is much smaller than $g$ and write with the Landau notation $f=o(g)$ or with the Hardy notation $f \ll g$ if $f(h) / g(h)$ tends to zero as $h$ tends to 0 . We will say that $f$ and $g$ are equivalent and write $f \sim g$ if $f(h) / g(h)$ tends to 1 as $h$ tends to 0 .

Proposition 3.5. 
1. Let $\ell=\ell_{x}+i \ell_{y} \in \mathcal{Q}$, with $\ell_{y} \leq \ell_{x}$. Then

(a) if $\ell_{y}<\ell_{x}$, the polynomial $t \mapsto q_{0}(\ell, t)$ has exactly one root in $] \mu,+\infty[$. Therefore $x \mapsto \mathscr{R}_{0}(\ell, x)$ has exactly one local extremum in $] \sqrt{\mu},+\infty[$, and this extremum is a minimum.

(b) If $\ell_{y}=\ell_{x} \leq \sqrt{3 \mu}$, the polynomial $t \mapsto q_{0}(\ell, t)$ has exactly one root in $\left[0,+\infty\left[\right.\right.$, which is equal to $\mu$. Therefore $x \mapsto \mathscr{R}_{0}(\ell, x)$ has exactly one local extremum in $\sqrt{\mu}$, and this extremum is a minimum. If $\ell_{y}=\ell_{x}=$ $\sqrt{3 \mu}$, it is a triple point.

(c) If $\ell_{y}=\ell_{x}>\sqrt{3 \mu}$, the polynomial $t \mapsto q_{0}(\ell, t)$ has three distinct roots in $\left[0,+\infty\left[, t_{1}(\ell)<t_{2}(\ell)=\mu<t_{3}(\ell)\right.\right.$. Therefore $x \mapsto \mathscr{R}_{0}(\ell, x)$ has exactly three local extrema in $[0,+\infty[$, and the only maximum point is $x_{2}(\ell)=\sqrt{t_{2}(\ell)}$.

2. In general, there exists $A_{0}>0$ such that for any $\ell \in \mathcal{Q}$ with $|\ell|^{2}>A_{0}$, the polynomial $t \mapsto q_{0}(\ell, t)$ has three positive well-separated roots,

$$
t_{1}(\ell) \sim \frac{\mu^{2}}{|\ell|^{2}} \ll t_{2}(\ell) \sim \mu \tau \ll t_{3}(\ell) \sim|\ell|^{2}-4 \mu \tau, \quad \text { with } \tau=\frac{\ell_{y}}{\ell_{x}} .
$$

$x_{2}(\ell)=\sqrt{t_{2}(\ell)}$ is the only maximum point of $x \mapsto \mathscr{R}_{0}(\ell, x)$. Defining $\omega_{2}(\ell)$ as the point on $\Gamma$ with abscissa $x_{2}(\ell), \theta_{2}(\ell)$ its argument, the precise following asymptotics hold

$$
t_{2}(\ell)=\mu \tau+4 \frac{\mu^{2}\left(\tau^{2}-1\right)}{|\ell|^{2}}+\mathcal{O}\left(\frac{1}{|\ell|^{4}}\right), \quad \theta_{2}=\frac{\pi}{2}-\phi+\mathcal{O}\left(\frac{1}{|\ell|^{2}}\right) .
$$

Furthermore the $t_{j}$ are continuous functions of $\ell$.

Remark 3.6. Since $\ell_{0}^{*} \in \mathscr{A}$ by Theorem 2.4, case 2 can happen for $\ell_{0}^{*}$ only if $\theta_{\min }>\frac{\pi}{4}$.

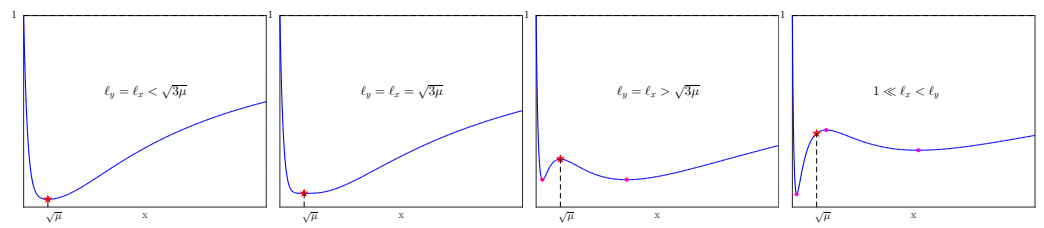

Fig. 3.2: Illustration of Proposition 3.5: variations of $x \mapsto \mathscr{R}_{0}(\ell, x)$. The red circles are the local extrema

Proof. The results of Proposition (3.5) are depicted on Fig. 3.2.

1. If $\ell_{y} \leq \ell_{x}$. Compute

$$
q_{0}(\ell, \mu)=\mu^{2}\left(2 \mu+|\ell|^{2}\right)\left(\ell_{y}-\ell_{x}\right) \leq 0 .
$$

(a) If $\ell_{y}<\ell_{x}, q_{0}(\ell, \mu)<0$, and since $t \mapsto q_{0}(\ell, t)$ tends to $+\infty$ as $t$ tends to $+\infty$, it has one or three roots in $] \mu,+\infty[$. Since the product of the roots is $\mu^{3} \frac{\ell_{y}}{\ell_{x}}<\mu^{3}$, there can not be three roots greater than $\mu$. Consequently $q_{0}(\ell, \cdot)$ has only one root $t_{3}(\ell)$ greater than $\mu$, and $x_{3}(\ell)=\sqrt{t_{3}(\ell)}$ is a minimum point of $\mathscr{R}_{0}(\ell, \cdot)$. 
(b) If $\ell_{y}=\ell_{x}$, then $t_{\text {min }}=\mu$ is a root of $q_{0}(\ell, \cdot)$. The other roots are the roots of the second degree polynomial

$$
P(t)=t^{2}+2\left(2 \mu-\ell_{x}^{2}\right) t+\mu^{2} .
$$

The reduced discriminant of $P$ is $\Delta=\left(\ell_{x}^{2}-\mu\right)\left(\ell_{x}^{2}-3 \mu\right)$. Therefore

- If $\sqrt{\mu}<\ell_{x}<\sqrt{3 \mu}$, the only root of $q_{0}(\ell, \cdot)$ is $t_{\min }=\mu$, hence $x_{\min }=\sqrt{\mu}$ is the only extremum point and it is a minimum point.

- If $\ell_{x} \leq \sqrt{\mu}, P$ has two negative roots. Since $P(0)=\mu^{2}>0$, $x_{\min }=\sqrt{\mu}$ is the only local extremum point on $[0,+\infty[$, and it is a minimum point.

- If $\ell_{x}=\sqrt{3 \mu}, \mu$ is a triple root of $q_{0}$. Again $x_{\text {min }}=\sqrt{\mu}$ is the only extremum point and it is a minimum point.

- If $\ell_{x}>\sqrt{3 \mu}, P$ has two distinct positive roots. Since $P(\mu)=$ $2 \mu\left(3 \mu-\ell_{x}^{2}\right)<0, t_{\min }=\mu$ is between these two roots, which are therefore minimum points, while $t_{\text {min }}$ is the only local maximum point.

2. In the general case, we need a perturbation analysis of the roots. Replacing $\ell_{y}$ by $\tau \ell_{x}$ in $q_{0}$, rewrite $q_{0}$ as

$$
q_{0}(\ell, t)=\ell_{x}\left(t^{3}+\left(3 \mu \tau-|\ell|^{2}\right) t^{2}+\mu\left(|\ell|^{2} \tau-3 \mu\right) t-\tau \mu^{3}\right),
$$

and use a small parameter $\varepsilon=\frac{1}{|\ell|^{2}}$ to define

$q_{0}(\ell, t)=\ell_{x}|\ell|^{2} \hat{q}_{0}\left(\frac{1}{|\ell|^{2}}, t, \tau\right), \hat{q}_{0}(\varepsilon, t, \tau):=-t^{2}+\mu \tau t+\varepsilon\left(t^{3}+3 \mu \tau t^{2}-3 \mu^{2} t-\tau \mu^{3}\right)$.

For any $\tau \in(0,+\infty), \hat{q}_{0}(\varepsilon, \cdot, \tau)$ is a perturbation of $P_{0}(t)=-t^{2}+\mu \tau t$, which has two simple roots $t_{1}=0$ and $t_{2}=\mu \tau$. By the implicit function theorem, there is $\left(\varepsilon_{0}>0, T_{1}, T_{2}>0\right)$ and two continuous functions $g_{j}$ : ]$-\varepsilon_{0}, \varepsilon_{0}\left[\times\left[\theta_{\min }, \theta_{\max }\right] \rightarrow\right] t_{j}-T_{j}, t_{j}+T_{j}\left[\right.$ such that for any $\varepsilon \leq \varepsilon_{0}$, for any $t \in] t_{j}-T_{j}, t_{j}+T_{j}\left[, \hat{q}_{0}(\varepsilon, t, \tau)=0 \Longleftrightarrow t=g_{j}(\varepsilon, \tau)\right.$. The functions $g_{j}$ are $\mathcal{C}^{\infty}$ as functions of $t$ in $]-\varepsilon_{0}, \varepsilon_{0}\left[\right.$. Then $A_{0}=1 / \sqrt{\varepsilon_{0}}$ is appropriate and $t_{j}(\ell)=g_{j}\left(\frac{1}{|\ell|^{2}}, \tau\right)$. A short computation gives the first terms in the Taylor expansion:

$$
t_{1}(\ell)=\mu^{2} \varepsilon+\mathcal{O}\left(\varepsilon^{2}\right), \quad t_{2}(\ell)=\mu \tau+4 \mu^{2}\left(\tau^{2}-1\right) \varepsilon+\mathcal{O}\left(\varepsilon^{2}\right) .
$$

Then there is a third real root, which can be obtained using the product of the roots equal to $\tau \mu^{3}$ :

$$
t_{3}(\ell)=\frac{\tau \mu^{3}}{t_{1}(\ell) t_{2}(\ell)}=\frac{1}{\varepsilon}(1+\mathcal{O}(\varepsilon))
$$

$x_{1}(\ell)=\sqrt{t_{1}(\ell)}$ and $x_{3}(\ell)=\sqrt{t_{3}(\ell)}$ are minimum points for $\mathscr{R}_{0}(\ell, \cdot)$, while $x_{2}(\ell)=\sqrt{t_{2}(\ell)}$ is the only maximum point. A short computation gives the next term in the expansion of $t_{2}(\ell)$. To prove the asymptotics on $\theta_{2}$, just notice that by notation 3.1 ,

$$
\tan \theta_{2} \sim \frac{1}{\tau}=\operatorname{cotan} \phi
$$

which implies that $\theta_{2} \sim \frac{\pi}{2}-\phi$. 
Theorem 3.7. Assume that $L=0$.

1. If $\theta_{\min } \leq \frac{\pi}{4}$, for any $k_{\max }<+\infty$, the solution $\left(\ell_{0}^{*}, \delta_{0}^{*}\right)$ to $(2.16)$ is given by

$$
\ell_{0}^{*}=\sqrt{\omega_{\min } \omega_{\max }}, \quad \delta_{0}^{*}=\left|\frac{\sqrt{\omega_{\max }}-\sqrt{\omega_{\min }}}{\sqrt{\omega_{\max }}+\sqrt{\omega_{\min }}}\right| .
$$

2. If $\frac{\pi}{4}<\theta_{\min }<\frac{\pi}{3}$, there exists $A>0$, for any $\omega_{\max }$ with $\left|\omega_{\max }\right|>A$, $\left(\ell_{0}^{*}, \delta_{0}^{*}\right)$ is given by (3.12).

In these two cases, $\omega_{\min }$ and $\omega_{\max }$ are the alternation points.

3. If $\theta_{\min } \geq \frac{\pi}{3}$, there exists $A>0$, for any $\omega_{\max }$ with $\left|\omega_{\max }\right|>A$, $\ell_{0}^{*}$ is the unique solution of the equation

$$
\ell=\sqrt{\omega_{2}(\ell) \omega_{\max }}
$$

where $x_{2}(\ell)=\sqrt{t_{2}(\ell)}$ is defined in Proposition 3.5, and $\omega_{2}(\ell)$ is the point on $\Gamma$ with real part $x_{2}(\ell)$. Then $\omega_{2}\left(\ell_{0}^{*}\right)$ and $\omega_{\max }$ are the alternation points.

Furthermore asymptotically we have

$$
\ell_{0}^{*} \sim \sqrt{2 \sqrt{\frac{\mu}{\sqrt{3}}} \omega_{\max }} e^{i \frac{\pi}{6}}, \delta_{0}^{*} \sim 1-\sqrt{2 \sqrt{3 \sqrt{3} \mu}} k_{\text {max }}^{-\frac{1}{2}}
$$

Proof. From notation 3.1, we find that

$$
\theta_{\min }=\frac{\pi}{4} \Longleftrightarrow t_{\min }=x_{\min }^{2}=\mu, \quad \theta_{\min }<\frac{\pi}{4} \Longleftrightarrow t_{\min }=x_{\min }^{2}>\mu .
$$

Case 1 If $\theta_{\min } \leq \frac{\pi}{4}$, define $\hat{\ell}=\sqrt{\omega_{\min } \omega_{\max }}$. Then $\operatorname{Arg} \hat{\ell}=\frac{1}{2}\left(\theta_{\min }+\theta_{\max }\right)$, therefore $\hat{\ell} \in \mathscr{A}$ and by Proposition 3.5.1.a,

$$
\sup _{\omega \in \Gamma} \mathscr{R}_{0}(\hat{\ell}, \omega)=\mathscr{R}_{0}\left(\hat{\ell}, \omega_{\min }\right)=\mathscr{R}_{0}\left(\hat{\ell}, \omega_{\max }\right) .
$$

Furthermore,

$$
V\left(\hat{\ell}, \omega_{\min }\right)=\frac{1}{\omega_{\max }-\omega_{\min }}=-V\left(\hat{\ell}, \omega_{\max }\right)
$$

which proves that $\omega_{\min }$ and $\omega_{\max }$ are alternation points, and Theorem 3.2 applies.

Case 2 If $\frac{\pi}{4}<\theta_{\min } \leq \frac{\pi}{3}$, define again $\hat{\ell}=\sqrt{\omega_{\min } \omega_{\max }}$. Since (3.16) is still holding, we are going to prove (3.15) for $\left|\omega_{\max }\right|$ large.

Note first that when $k_{\max }$ tends to infinity, $k_{\max }$ and $\left|\omega_{\max }\right|$ are equivalent, and $\theta_{\max }$ tends to zero .

By definition of $\hat{\ell}$ we have

$$
|\hat{\ell}|^{2} \approx\left|\omega_{\max }\right|, \operatorname{Arg} \hat{\ell}=\frac{1}{2}\left(\theta_{\min }+\theta_{\max }\right) \sim \frac{1}{2} \theta_{\min }, \hat{\tau} \sim \tan \frac{1}{2}\left(\theta_{\min }+\theta_{\max }\right) .
$$

There exists $B>0$ such that for $\left|\omega_{\max }\right|>B,|\hat{\ell}|^{2}>A_{0}$ defined in Lemma 3.5, and by Proposition 3.5.2, $q_{0}(\hat{\ell}, \cdot)$ has three distinct roots, among them only $t_{2}(\hat{\ell})$ is a maximum point. From the asymptotics in (3.9), for all $\varepsilon>0$, there exists $C>B$ such that for $\left|\omega_{\max }\right|>C,|\hat{\ell}|^{2}>A_{0}$ and $\left.t_{2}(\hat{\ell}) \in\right] \mu \hat{\tau}-\varepsilon, \mu \hat{\tau}+\varepsilon[$. 
We need now to compare $t_{\min }$ and $t_{2}(\hat{\ell})$. Start with the comparison of $t_{\min }$ and $\mu \hat{\tau}$ :

$$
\frac{\mu \hat{\tau}}{t_{\min }}=\tan \theta_{\min } \tan \frac{\theta_{\min }+\theta_{\max }}{2} .
$$

Since $\theta_{\min }<\frac{\pi}{3}$, this quantity is strictly smaller than 1 for $\theta_{\max }=0$. Therefore for any $\varepsilon^{\prime}$, there exists $A>C$ such that

$$
\left|\omega_{\max }\right|>A \Longrightarrow \tan \theta_{\min } \tan \frac{\theta_{\min }+\theta_{\max }}{2}<1-\varepsilon^{\prime},
$$

or equivalently $\mu \hat{\tau}<t_{\min }-\varepsilon^{\prime} t_{\min }$. Collecting these informations, for any $\varepsilon$ and $\varepsilon^{\prime}$, there exists $A>0$ such that for $\left|\omega_{\max }\right|>A$,

$$
t_{2}(\hat{\ell})<\mu \hat{\tau}+\varepsilon<t_{\text {min }}-\varepsilon^{\prime} t_{\text {min }}+\varepsilon .
$$

Choosing $\varepsilon^{\prime} t_{\text {min }}=2 \varepsilon$ yields $t_{2}(\hat{\ell})<t_{\text {min }}-\varepsilon<t_{\text {min }}$. Therefore on the interval $\left[x_{\min }, x_{\max }\right]$, the maximum points of $\mathscr{R}(\hat{\ell}, \cdot)$ are $x_{\min }$ and $x_{\max }$. At these points there is equioscillation and (3.15) is proved.

Then Theorem 3.2 applies and proves the result.

Case 3 If $\theta_{\min } \geq \frac{\pi}{3}$, the proof has several steps.

Step 1 For a given $k_{\min }$, show that $\ell_{0}^{*}$ tends to infinity as $k_{\max }$ tends to infinity:

$$
\forall A>0, \exists B>0, \forall k_{\max }>B,\left|\ell_{0}^{*}\right|^{2}>A .
$$

Step 2 For large $k_{\max }$, show that equation (3.13) has a solution $\hat{\ell}$. More precisely by Proposition 3.5, Case 2, introduce the function

$$
\Psi(\ell)=\frac{\ell^{2}}{\omega_{2}(\ell)},
$$

and show by perturbation and homotopy arguments that the equation

$$
\Psi(\ell)=\omega_{\max }
$$

has a solution.

Step 3 Show that $\omega_{2}(\hat{\ell})$ and $\omega_{\max }$ are alternation points for $\hat{\ell}$, see Definition 3.2. Conclude by Theorem 3.2 that $\hat{\ell}=\ell_{0}^{*}$.

Step 4 Perform the asymptotics on (3.18).

Step 1 To emphasize the dependance of $\ell_{0}^{*}$ in $k_{\max }$, define the continuous function $\ell_{0}^{*}\left(k_{\max }\right)$. Suppose by contradiction that

$$
\exists C>0, \forall B>0, \exists k_{\max }>B,\left|\ell_{0}^{*}\left(k_{\max }\right)\right| \leq C .
$$

For any $\ell$, since $x \mapsto q_{0}\left(\ell, x^{2}\right)$ tends to infinity at infinity, there exists $D(\ell)$ such that $q_{0}\left(\ell, x^{2}\right)$ is positive in $[D(\ell),+\infty]$. By compactness, there exists $X$ such that for any $\ell$ in the ball of radius $C, q_{0}\left(\ell, x^{2}\right)$ is positive in $[X,+\infty]$.

By continuity and compactness, $\sup _{\ell \in \mathscr{A},|\ell| \leq C, x \in\left[x_{\min }, X\right]} \mathscr{R}(\ell, x)=D<1$. Since its derivative is positive, the function $x \rightarrow \mathscr{R}\left(\ell_{0}^{*}\left(k_{\max }\right), x\right)$ is strictly increasing in $(X,+\infty)$. For $\ell$ and $\omega$ in $\mathscr{A}$, the argument of $\ell / \omega$ is between 0 and $\pi / 2$, therefore its real part is positive. Compute then

$$
\mathscr{R}_{0}(\ell, \omega)=\left|\frac{1-\frac{\ell}{\omega}}{1+\frac{\ell}{\omega}}\right|^{2}=1-\frac{4 \operatorname{Re} \frac{\ell}{\omega}}{\left|1+\frac{\ell}{\omega}\right|^{2}} \geq 1-4 \operatorname{Re} \frac{\ell}{\omega} \geq 1-4\left|\frac{\ell}{\omega}\right| .
$$


Apply this lower bound to $\omega_{\max }$ and $\ell_{0}^{*}\left(k_{\max }\right)$ to obtain

$$
\mathscr{R}_{0}\left(\ell_{0}^{*}\left(k_{\max }\right), \omega_{\max }\right) \geq 1-\frac{C}{\sqrt{\left|B^{2}+\alpha\right|}} .
$$

Choose now $B$ such that $1-\frac{C}{\sqrt{\left|B^{2}+\alpha\right|}}>D$. Then $\mathscr{R}\left(\ell_{0}^{*}\left(k_{\max }\right), x\right)$ is bounded by $D$ on $\left[x_{\min }, X\right]$, increasing on $\left[X, x_{\max }\right]$, and $\mathscr{R}\left(\ell_{0}^{*}\left(k_{\max }\right), x_{\max }\right)>D$. Therefore $\omega_{\max }$ is a strict maximum point. This is in contradiction with Theorem 2.5, which asserts that $\omega \mapsto \mathscr{R}_{0}\left(\ell_{0}^{*}\left(k_{\max }\right), \omega\right)$ equioscillates in at least two points, concluding the proof of Step 1.

Step 2 Choose as $A$ the $A_{0}$ from Proposition 3.5. By Step 1, choose $B$ from $A$, then for any $k_{\max }>B, \ell_{0}^{*} \in \mathscr{B}(A)$, where $\mathscr{B}(A)=\left\{\ell \in \mathscr{A},|\ell|^{2}>A\right\}$. Furthermore, for any $\ell$ in $\mathscr{B}(A)$, there is one and only one local maximum point $\omega_{2}(\ell)$ on $\Gamma$, with abscissa $x_{2}(\ell)=\sqrt{t_{2}(\ell)}$. Since the real part of $\ell$ is positive, $(3.13)$ is equivalent to solving $(3.18,3.19) . x_{2}$ is a continuous function of $\ell$ in $\mathscr{B}(A)$. By the implicit functions theorem used recursively, it is a $\mathcal{C}^{\infty}$ function in $\mathscr{B}(A)$, and so are $\omega_{2}$ and $\Psi$. From $(3.9,3.10)$, we find an equivalent to the point $\omega_{2}(\ell)$ on $\Gamma$, uniform in $\tau$ in a compact interval: there exists $C>0$ such that for any $\ell$ in $\mathscr{B}(A)$,

$$
\left|\omega_{2}(\ell)-i \sqrt{\mu \frac{\tau^{2}+1}{\tau}} e^{-i \phi}\right| \leq \frac{C}{|\ell|^{2}} .
$$

Write $\ell=\ell_{x} \sqrt{1+\tau^{2}} e^{i \phi}$, with $\tau=\tan \phi$,

$$
\frac{\ell^{2}}{\omega_{2}(\ell)} \sim-i \ell_{x}^{2} \sqrt{\frac{\tau\left(\tau^{2}+1\right)}{\mu}} e^{3 i \phi},
$$

and we can easily estimate the remainder with $C^{\prime}=\frac{C}{2 \mu}$. Define

$$
\begin{gathered}
Z\left(\phi, \ell_{x}^{2}\right)=\Psi(\ell), \quad z(\phi)=-i \sqrt{\frac{\tan \phi\left(\tan ^{2} \phi+1\right)}{\mu}} e^{3 i \phi}, \\
\left|Z\left(\phi, \ell_{x}^{2}\right)-\ell_{x}^{2} z(\phi)\right| \leq C^{\prime}
\end{gathered}
$$

The variations of $z$ in $\phi$ are plotted in Figure 3.3. All properties described in the graph can be obtained by the analytical study in parametric form in $\phi$.

The maximum of $\operatorname{Re} z$ is obtained at point $z\left(\phi_{M}\right)$ ( square magenta in Figure 3.3), with $\frac{\pi}{6}<\phi_{M}<\frac{\pi}{4}$.

For $\ell \in \mathscr{B}(A), \phi \mapsto Z\left(\phi, \ell_{x}\right)$ follows a $\mathcal{C}^{1}$ curve, contained in the tube $\mathcal{T}\left(\ell_{x}\right)$ of width $C^{\prime}$ centered on $\ell_{x}^{2} G$.

Let $\omega_{\max }$ in the strict exterior right of $\mathcal{T}(A)$, and $A^{\prime}>A$, such that $\omega_{\max }$ is the strict exterior left of $\mathcal{T}\left(A^{\prime}\right)$ (see Figure 3.4). This is obtained when

$$
A \operatorname{Re} z\left(\phi_{M}\right)+C^{\prime}<\operatorname{Re} \omega_{\max }<A^{\prime} \operatorname{Re} z\left(\phi_{M}\right)-C^{\prime}, \operatorname{Im} \omega_{\max }<A^{\prime} \operatorname{Im} z\left(\phi_{M}\right) .
$$

From $\omega_{\max }=\sqrt{\alpha+k_{\max }^{2}+2 i \mu}$, we obtain that $\operatorname{Re} \omega_{\max }=\mathcal{O}\left(k_{\max }\right)$ and $\operatorname{Im} \omega_{\max }=\mathcal{O}\left(k_{\max }^{-1}\right)$, which shows that the previous inequalities define a range 


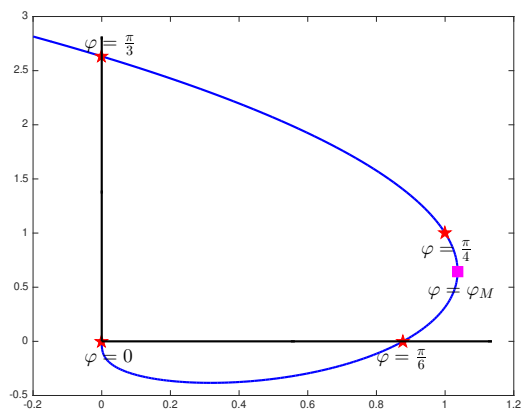

Fig. 3.3: The curve $G$ of Variations of $z(\phi)$ for $\mu=1$

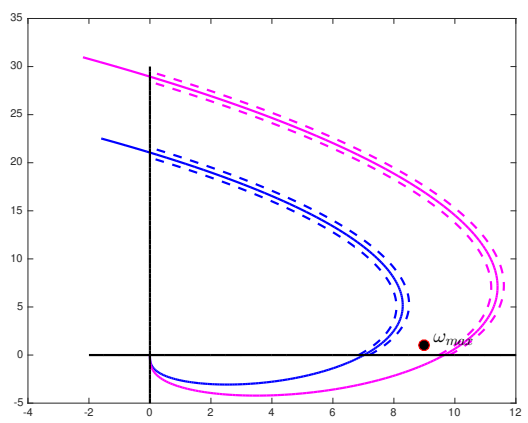

Fig. 3.4: $A z$ together with the tube containing $Z(\cdot, \sqrt{A})$ for $A$ and $A^{\prime}$

for $k_{\max }$, and then a range for $A^{\prime}$. The arguments below use complex analysis, see [38]. Define the contour $\gamma_{z}\left(A, A^{\prime}\right)$ as follows: run $A^{\prime} G$ from 0 to $\frac{\pi}{3}$, then the imaginary axis backward until it meets $A G$, then run back to the origin along $A G$. Similarly define $\gamma_{Z}\left(A, A^{\prime}\right)$ by running $Z\left(\phi, \sqrt{A^{\prime}}\right)$ from 0 to $\frac{\pi}{3}$, then the imaginary axis backward until it meets $Z(\phi, \sqrt{A})$, then run back to the origin along $Z(\phi, \sqrt{A})$. This contour can be defined by continuity when $A=A^{\prime}: \gamma_{Z}\left(A^{\prime}, A^{\prime}\right)$ is the contour obtained by taking the limit as $A$ tends to $A^{\prime}$ of $\gamma_{Z}\left(A, A^{\prime}\right)$, and is just running forth and back along $Z\left(\phi, \sqrt{A^{\prime}}\right)$.

The index of a contour $\gamma$ about $\omega_{\max }$ is denoted $\operatorname{Ind}\left(\gamma, \omega_{\max }\right)$, it gives the number of times $\gamma$ curve passes (counterclockwise) around a point. By hypothesis, $\operatorname{Ind}\left(\gamma_{z}\left(A, A^{\prime}\right), \omega_{\max }\right)=1$. By homotopy in the tubes, $\operatorname{Ind}\left(\gamma_{Z}\left(A, A^{\prime}\right), \omega_{\max }\right)=$ 1 as well.

Consider now the function $\ell_{x}^{2} \mapsto \gamma_{Z}\left(A, \ell_{x}^{2}\right)$ for $\ell_{x}^{2} \in\left(A, A^{\prime}\right)$. It is a continuous function, and therefore must preserve the index as long as the contour does not meet $\omega_{\max }$. But since $\operatorname{Ind}\left(\gamma_{Z}\left(A, A^{\prime}\right), \omega_{\max }\right)=1$ while $\operatorname{Ind}\left(\gamma_{Z}\left(A^{\prime}, A^{\prime}\right), \omega_{\max }\right)=$ 0 , there is a value of $\ell_{x}$ for which $\omega_{\max } \in Z\left(\phi, \ell_{x}\right)$, that is a value of $\ell=$ $\ell_{x}(1+i \tan \phi)$ for which $\omega_{\max }=Z\left(\phi, \ell_{x}\right)$. Then define $\hat{\ell}$ to be this value, that is

$$
\hat{\ell}=\sqrt{\omega_{2}(\hat{\ell}) \omega_{\max }}
$$


Step 3 By Proposition 3.5, we know that

$$
\sup _{\omega \in \Gamma} \delta_{0}(\hat{\ell}, \omega)=\max \left(\delta_{0}\left(\hat{\ell}, \omega_{2}(\hat{\ell})\right), \delta_{0}\left(\hat{\ell}, \omega_{\max }\right)\right) .
$$

Moreover we deduce from (3.22) that

$$
\delta_{0}\left(\hat{\ell}, \omega_{2}(\hat{\ell})\right)=\delta_{0}\left(\hat{\ell}, \omega_{\max }\right)=\left|\frac{\sqrt{\omega_{2}(\hat{\ell})}-\sqrt{\omega_{\max }}}{\sqrt{\omega_{2}(\hat{\ell})}+\sqrt{\omega_{\max }}}\right| .
$$

Therefore the equioscillation property is fulfilled:

$$
\sup _{\omega \in \Gamma} \delta_{0}(\hat{\ell}, \omega)=\delta_{0}\left(\hat{\ell}, \omega_{2}(\hat{\ell})\right)=\delta_{0}\left(\hat{\ell}, \omega_{\max }\right) .
$$

Now since

$$
V\left(\hat{\ell}, \omega_{\max }\right)=\frac{1}{\omega_{2}(\hat{\ell})-\omega_{\max }}=-V\left(\hat{\ell}, \omega_{2}(\hat{\ell})\right),
$$

$\omega_{\max }$ and $\omega_{2}(\hat{\ell})$ are alternation points and Theorem 3.2 applies, to see that $\hat{\ell}$ is a strict local minimum, therefore coincides with $\ell_{0}^{*}$.

Step 4 Note the principal approximations: $\hat{\ell}_{0}$ for $\hat{\ell}, \hat{\phi}_{0}$ for the argument of $\hat{\ell}, \hat{\tau}_{0}=\tan \hat{\phi}_{0}, \hat{\theta}_{0}$ the argument of $\omega_{2}\left(\hat{\ell}_{0}\right)$. Consider the arguments in (3.22).

By Proposition 3.5, since $\arg \omega_{\max } \sim 0$, we obtain

$$
\hat{\phi}_{0}=\frac{1}{2} \hat{\theta}_{0}=\frac{1}{2}\left(\frac{\pi}{2}-\hat{\phi}_{0}\right) \Longrightarrow \hat{\phi}_{0}=\frac{\pi}{6}, \hat{\theta}_{0}=\frac{\pi}{3}, \hat{\tau}_{0}=\frac{1}{\sqrt{3}} .
$$

Now from the approximation of $\operatorname{Re} \omega_{2}(\hat{\ell})$ in the proposition, we find

$$
\left|\omega_{2}\left(\hat{\ell}_{0}\right)\right|^{2}=\mu\left(\hat{\tau}_{0}+\frac{1}{\hat{\tau}_{0}}\right)=4 \mu \hat{\tau}_{0}
$$

Therefore $\omega_{2}\left(\hat{\ell}_{0}\right)=\sqrt{4 \mu \hat{\tau}_{0}} e^{i \frac{\pi}{3}}$, and

$$
\hat{\ell}_{0}=\sqrt{\sqrt{4 \mu \hat{\tau}_{0}} \omega_{\max }} e^{i \frac{\pi}{6}} .
$$

Furthermore,

$$
\delta_{0}\left(\hat{\ell}, \omega_{\max }\right) \sim 1-2 \operatorname{Re} \frac{\hat{\ell}_{0}}{\omega_{\max }} \sim 1-\sqrt[4]{4 \mu \hat{\tau}_{0}} k_{\max }^{-\frac{1}{2}} .
$$

Remark 3.8. In other contexts (real elliptic equations, advection-diffusion equations), where the optimal parameter is real for symmetry reasons, alternation suffices to define the parameter, and from this equation deduce the value of the parameter as solution of an algebraic equation. Because of the complex coefficients, the situation here is very different, even though the final formula is the same in some cases as in the elliptic case. Equioscillation at endpoints defines a bounded closed curve $\mathcal{C}$ in $\mathscr{A}$. The optimal parameter is the point of $\mathcal{C}$ which realizes the least value of the convergence factor at the endpoints of the interval. 
3.4. The overlapping case. By Theorems 2.8 and 2.4, the best solution $\ell_{L}^{*}$ exists and is unique, and belongs to $\mathscr{A}$.

In order to characterize this solution, we first need to identify the extremal points of $\mathscr{R}_{L}(\ell, \cdot)$. They are either endpoints of the interval, or local extremum points. The local extremum points are the roots of the real fourth order polynomial $q_{L}$, which are functions of $\ell$ and $L$.

Remark 3.9. For positive $L$, the polynomial $q_{L}$ is of degree 4 , and its roots can be obtained by radicals, using the Ferro/Tartaglia/Cardano formulas [10]. However these formulas are even less tractable than in the case $L=0$, see Remark 3.3.

Proposition 3.10 analyses $q_{L}$ and gives asymptotics of the roots. These asymptotic results involve two scales, $\ell_{x}^{-2}$ and $L \ell_{x}$, and therefore it is convenient to introduce the family of sets

$$
\mathscr{B}(A, \gamma)=\left\{(\ell, L) \in \mathcal{A} \times \mathbb{R}_{+}^{*}, A<\ell_{x}^{2} \text { and } L \ell_{x}<\gamma\right\} .
$$

Then in Proposition 3.11, the points where a local maximum of $\mathscr{R}_{L}(\ell, \cdot)$ is reached are identified.

By Theorem $2.8 \mathscr{R}_{L}\left(\ell_{L}^{*}, \cdot\right)$ equioscillates in at least two distinct points, therefore we first define a set of parameters $\ell$ for which $\mathscr{R}_{L}(\ell, \cdot)$ equioscillates at the points identified in Proposition 3.11. This set is called $\mathcal{C}_{L}$. Then $\hat{\ell}$ is defined as a minimum point of $\mathscr{R}_{L}(\ell, \cdot)$ along $\mathcal{C}_{L}$, using a Lagrange multiplier, and asymptotic expansions for $\hat{\ell}$ and $\max _{x} \mathscr{R}_{L}(\hat{\ell}, x)$ are provided.

The last step relies on the alternation theorem 3.2, proving that $\hat{\ell}$ is indeed a strict minimum point for $h_{L}$.

Proposition 3.10. There exists $\left(A_{0}, \gamma_{0}\right)$ with $A_{0}>3 \mu$ and $0<\gamma_{0}<\frac{2}{A_{0}\left(1+\tan ^{2} \theta_{\min }\right)}$ such that for any $(\ell, L) \in \mathscr{B}\left(A_{0}, \gamma_{0}\right)$, the polynomial $q_{L}(\ell, t)$ defined in $(3.3)$ has exactly 4 distincts positive roots $t_{i}(L, \ell)$. These roots are continuous functions of $L$ and $\ell$, and they behave asymptotically as follows:

$$
t_{1}(L, \ell) \sim \frac{\mu^{2}}{|\ell|^{2}} \ll t_{2}(L, \ell) \sim \mu \tau \ll t_{3}(L, \ell) \sim|\ell|^{2} \ll t_{4}(L, \ell) \sim \frac{2 \ell_{x}}{L},
$$

where $\tau=\frac{\ell_{y}}{\ell_{x}}$, and $\sim$ means $\mathcal{O}\left(L \ell_{x}\right)+\mathcal{O}\left(\frac{1}{|\ell|^{2}}\right)$. Define $x_{j}(L, \ell)=\sqrt{t_{j}(L, \ell)}$. Then $x_{1}(L, \ell)$ and $x_{3}(L, \ell)$ are local minimum points, while $x_{2}(L, \ell)$ and $x_{4}(L, \ell)$ are local maximum points for the function $x \mapsto \mathscr{R}_{L}(\ell, x)$. More precisely,

1. If $\ell_{y} \leq \ell_{x}$, the polynomial $q_{L}$ has exactly two roots $t_{3}(\ell, L)$ and $t_{4}(\ell, L)$ in $] \mu,+\infty\left[\right.$. Therefore the only local maximum point of $x \mapsto \mathscr{R}_{L}(\ell, x)$ in ]$\sqrt{\mu},+\infty\left[\right.$ is $x_{4}(\ell, L)=\sqrt{t_{4}(\ell, L)}$.

2. If $\ell_{y}>\ell_{x}$, the function $x \mapsto \mathscr{R}_{L}(\ell, x)$ has three local extrema in $] \sqrt{\mu},+\infty[$. $x_{2}(\ell, L)=\sqrt{t_{2}(\ell, L)}$ and $x_{4}(\ell, L)=\sqrt{t_{4}(\ell, L)}$ are the maximum points.

Furthermore for any $\varepsilon>0$, there exists $\left(A_{0}, \gamma_{0}\right)$ such that for any $(\ell, L) \in \mathscr{B}\left(A_{0}, \gamma_{0}\right)$ with $\ell_{y}<\ell_{x},-\varepsilon<t_{2}(L, \ell)-\mu \tau<0$.

Proof. We will write the polynomial $q_{L}$ in (3.3) with two small parameters $\varepsilon=\frac{1}{\ell_{x}^{2}}$ and $\gamma=L \ell_{x} . \tau=\frac{\ell_{y}}{\ell_{x}}$ is fixed. Start with

$$
q_{L}(\ell, t)=q_{0}(\ell, t)+L \tilde{q}_{0}(\ell, t) .
$$

The properties of $q_{0}$ have been set in Proposition 3.5. The case $1 \mathrm{~b}$ in Proposition 3.5 is excluded by choosing $A_{0}>3 \mu$. In the other cases, the roots are well-separated, and 
(3.9) holds for some $A_{0}$ and $\ell_{x}^{2}>A_{0}$. Rewrite $q_{L}$ by hightlighting the parameters $\varepsilon$ and $\gamma$ :

$$
\begin{aligned}
& q_{L}(\ell, t)=\ell_{x}|\ell|^{2} \hat{q}_{L}\left(\frac{1}{\ell_{x}^{2}}, L \ell_{x}, t, \tau\right) \\
& \hat{q}_{L}(\varepsilon, \gamma, t, \tau)=\hat{q}_{0}\left(\frac{\varepsilon}{1+\tau^{2}}, t, \tau\right)+\frac{\gamma}{1+\tau^{2}} \hat{q}_{1}(\varepsilon, t, \tau) \\
& \hat{q}_{0}(\varepsilon, t, \tau)=-t^{2}+\mu \tau t+\varepsilon\left(t^{3}+3 \mu \tau t^{2}-3 \mu^{2} t-\tau \mu^{3}\right) \\
& \hat{q}_{1}(\varepsilon, t, \tau)=-\frac{1}{2}\left(1+\tau^{2}\right)^{2} t^{2}+\varepsilon^{2} t\left(\left(1-\tau^{2}\right) t^{2}+4 \mu \tau t-\mu^{2}\left(1-\tau^{2}\right)\right) \\
& \quad+\varepsilon^{4}\left(-\frac{1}{2} t^{4}+\mu^{2} t^{2}-\frac{1}{2} \mu^{4}\right)
\end{aligned}
$$

The analysis of the roots of $q_{L}$ thus appears as a perturbation problem in two parameters, $\varepsilon=\frac{1}{\ell_{x}^{2}}$ and $\gamma=L \ell_{x}$.

By the implicit function theorem starting from $q_{0}$, there exists $\left(A_{0}, \gamma_{0}\right)$ such that for any $\tau$, for $(\ell, L) \in \mathscr{B}\left(A_{0}, \gamma_{0}\right), t \mapsto \hat{q}_{L}(\ell, t)$ has three distinct positive roots, continuous in $\ell$ and $L, t_{j}(\ell, L)$ such that $t_{j}(\ell, 0)=t_{j}(\ell)$. There is a fourth root, $t_{4}(\ell, L)$, obtained by considering the sum of the roots of $\hat{q}_{L}$ and taking the asymptotics. For large $\ell$, since $t_{3}(\ell, L) \sim|\ell|^{2}$,

$$
\sum_{i=1}^{4} t_{i}(\ell, L)=\frac{2 \ell_{x}}{L}\left(1+L \ell_{x}\left(1-\tau^{2}\right)\right) \gg|\ell|^{2} \sim \sum_{i=1}^{3} t_{i}(\ell, L) \text { since } L \ell_{x} \ll 1 .
$$

From this we conclude that $t_{4}(\ell, L) \sim \frac{2 \ell_{x}}{L}$, and all the roots are well-separated. One extra term can be computed in the asymptotic behavior of $t_{2}(\ell, L)$. It is obtained from $t_{2}(\ell)$ by Taylor expansion, given by $t_{2}(\ell)+C_{2}(\ell, \varepsilon) \gamma+\mathcal{O}\left(\gamma^{2}\right)$, with

$$
C_{2}(\ell, \varepsilon)=-\frac{\frac{1}{1+\tau^{2}} \hat{q}_{1}\left(\varepsilon, t_{2}(\ell), \tau\right)}{\frac{d}{d t} \hat{q}_{0}\left(\frac{\varepsilon}{1+\tau^{2}}, t_{2}(\ell), \tau\right)}
$$

By continuity, $C_{2}(\ell, \varepsilon)=C_{2}(\ell, 0)+\mathcal{O}(\varepsilon)$, and we only need to compute the latter, that is

$$
C_{2}(\ell, 0)=-\frac{\frac{1}{1+\tau^{2}}\left(-\frac{1}{2}\left(1+\tau^{2}\right)^{2} t_{2}(\ell)^{2}\right)}{\mu \tau-2 t_{2}(\ell)}=\frac{1}{2} \frac{\left(1+\tau^{2}\right) t_{2}(\ell)^{2}}{\mu \tau-2 t_{2}(\ell)} .
$$

Since $t_{2}(\ell) \sim \mu \tau+4 \mu^{2}\left(\tau^{2}-1\right) \varepsilon$, we obtain

$$
t_{2}(L, \ell) \sim \mu \tau+4 \mu^{2}\left(\tau^{2}-1\right) \varepsilon-\frac{\mu \tau}{2}\left(1+\tau^{2}\right) \gamma .
$$

Up to now, we have not used the fact that $\ell \in \mathscr{A}$. This property imposes that $\tau \in\left[0, \tan \theta_{\text {min }}\right]$, and we can obtain uniform bounds in $\tau$ for the roots. Therefore for all $\varepsilon>0$, upon reducing $\gamma_{0}$ and increasing $A_{0}$, we can have for any $\ell$ in $\mathscr{B}\left(\gamma_{0}, A_{0}\right)$, if $\ell_{y}<\ell_{x},-\varepsilon<t_{2}(L, \ell)-\mu \tau<0$.

Proposition 3.11. Let $\left(A_{0}, \gamma_{0}\right)$ such that Proposition 3.10 holds.

1. If $\theta_{\min } \leq \frac{\pi}{4}\left(x_{\min } \geq \sqrt{\mu}\right)$, then for any $(\ell, L) \in \mathscr{B}\left(A_{0}, \gamma_{0}\right)$,

$$
\sup _{x \in\left[x_{\min },+\infty\right)} \mathscr{R}_{L}(\ell, x)=\max \left(\mathscr{R}_{L}\left(\ell, x_{\text {min }}\right), \mathscr{R}_{L}\left(\ell, x_{4}(L, \ell)\right)\right),
$$

with $x_{\min }=\sqrt{\mu}$ if $\theta_{\min }=\frac{\pi}{4}$. 


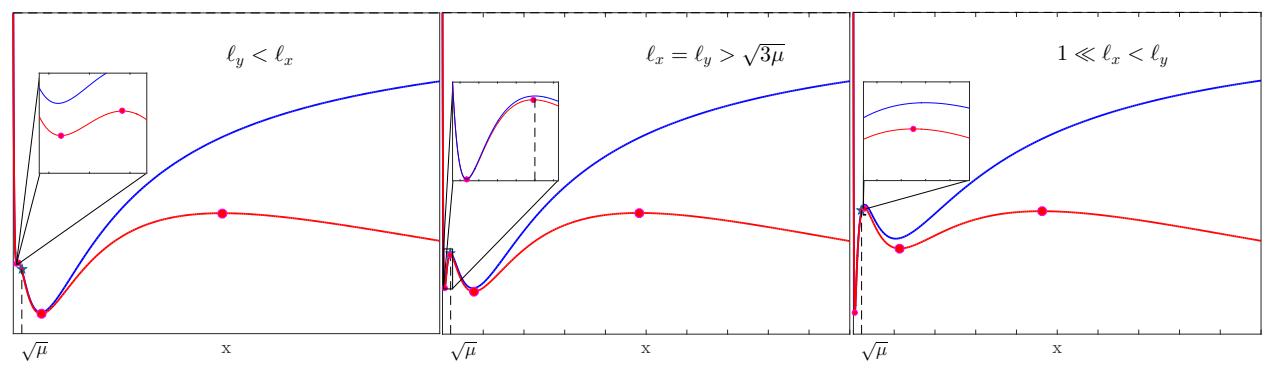

Fig. 3.5: Illustration of Proposition 3.10: variations of $x \mapsto \mathscr{R}_{L}(\ell, x)$. The red circles are the local extrema

2. If $\theta_{\min }>\frac{\pi}{4}\left(x_{\min }<\sqrt{\mu}\right)$, then for any $(\ell, L) \in \mathscr{B}\left(A_{0}, \gamma_{0}\right)$,

- If $\ell_{y}<\ell_{x}$, and $x_{2}(L, \ell)<x_{\min }$, then

$$
\sup _{x \in\left[x_{\min },+\infty\right)} \mathscr{R}_{L}(\ell, x)=\max \left(\mathscr{R}_{L}\left(\ell, x_{\min }\right), \mathscr{R}_{L}\left(\ell, x_{4}(L, \ell)\right)\right) .
$$

- In any other case, $x_{2}(L, \ell) \geq x_{\min }$ and

$$
\sup _{x \in\left[x_{\min },+\infty\right)} \mathscr{R}_{L}(\ell, x)=\max \left(\mathscr{R}_{L}\left(\ell, x_{2}(L, \ell)\right), \mathscr{R}_{L}\left(\ell, x_{4}(L, \ell)\right)\right) .
$$

Furthermore

$$
\begin{aligned}
& \mathscr{R}_{L}\left(\ell, x_{\min }\right) \sim 1-4 \operatorname{Re} \frac{\omega_{\min }}{\ell}, \quad \mathscr{R}_{L}\left(\ell, x_{2}(L, \ell)\right) \sim 1-4 \operatorname{Re} \frac{\omega_{2}}{\ell}, \\
& \mathscr{R}_{L}\left(\ell, x_{4}(L, \ell)\right) \sim 1-4 \sqrt{2 \ell_{x} L} .
\end{aligned}
$$

Proof. by Proposition 3.10, there exists $\left(A_{0}, \gamma_{0}\right)$ such that for any $(\ell, L) \in \mathscr{B}\left(A_{0}, \gamma_{0}\right)$, the polynomial $q_{L}(\ell, t)$ defined in (3.3) has exactly 4 distincts positive roots $t_{i}(L, \ell)$ with a precise asymptotic behavior. Note

$$
x_{j}(L, \ell)=\sqrt{t_{j}(L, \ell)}, \quad y_{j}(L, \ell)=\frac{\mu}{x_{j}(L, \ell)}, \quad \omega_{j}(L, \ell)=x_{j}(L, \ell)+i y_{j}(L, \ell) .
$$

1. If $\theta_{\min } \leq \frac{\pi}{4}$, since $\mathscr{B}\left(A_{0}, \gamma_{0}\right) \subset \mathscr{A}$, we have $\ell_{y} \leq \ell_{x}$, or equivalently $\tau \leq 1$, and

$$
x_{1}(L, \ell) \ll x_{2}(L, \ell) \leq \sqrt{\mu} \leq x_{\min } \ll x_{3}(L, \ell) \ll x_{4}(L, \ell) .
$$

Therefore the only local maximum point in $\left[x_{\min },+\infty\right)$ is $x_{4}(L, \ell)$ and (3.28) is proved.

2. If $\theta_{\min }>\frac{\pi}{4}$, then $x_{\min }<\sqrt{\mu}$ and $x_{2} \sim \sqrt{\mu \tau}$. Therefore

- If $\ell_{y}<\ell_{x}$, then $\tau<1$, and

$$
x_{1}(L, \ell) \ll x_{\min } \bar{\sim} x_{2}(L, \ell)<\sqrt{\mu} \ll x_{3}(L, \ell) \ll x_{4}(L, \ell) .
$$

- If $x_{2}(L, \ell)<x_{\text {min }}$, then the local maximum point for $\mathscr{R}_{L}(\ell, x)$ on $\left(x_{\min },+\infty\right)$ is $x_{4}(L, \ell)$, which proves $(3.28)$.

- If $x_{2}(L, \ell) \geq x_{\min }$, then the local maximum points for $\mathscr{R}_{L}(\ell, x)$ on $\left(x_{\min },+\infty\right)$ are $x_{j}(L, \ell)$ for $j=2,4$ and (3.29) is proved. 
- If $\ell_{y}=\ell_{x}$, then

$$
x_{1}(L, \ell) \ll x_{\min }<x_{2}(L, \ell)=\sqrt{\mu} \ll x_{3}(L, \ell) \ll x_{4}(L, \ell),
$$

and (3.29) is proved.

- If $\ell_{y}>\ell_{x}$, then

$$
x_{1}(L, \ell) \ll x_{\min }<\sqrt{\mu}<x_{2}(L, \ell) \ll x_{3}(L, \ell) \ll x_{4}(L, \ell) .
$$

The local maximum points for $\mathscr{R}_{L}(\ell, x)$ on $(0,+\infty)$ are $x_{j}(L, \ell)$ for $j=$ 2,4 and (3.29) is proved.

Compute now the asymptotics of the convergence factors. It is easy to see that $\omega_{4}(L, \ell) \sim x_{4}(L, \ell) \sim \sqrt{\frac{2 \ell_{x}}{L}} \gg 1$. Since

$$
\frac{\ell}{\omega_{4}} \sim \frac{\ell}{x_{4}} \sim(1+i \tau) \sqrt{\frac{L \ell_{x}}{2}}
$$

then

$$
\mathscr{R}_{0}\left(\ell, x_{4}(L, \ell)\right) \sim 1-4 \frac{\ell_{x}}{x_{4}(L, \ell)} \sim 1-2 \sqrt{2 L \ell_{x}}, \quad e^{-2 L x_{4}(L, \ell)} \sim 1-2 \sqrt{2 L \ell_{x}},
$$

which together gives

$$
\mathscr{R}_{L}\left(\ell, x_{4}(L, \ell)\right) \sim 1-4 \sqrt{2 L \ell_{x}} .
$$

Compute now for $x=\mathcal{O}(1)$,

$$
\mathscr{R}_{L}(\ell, x) \sim 1-4 \operatorname{Re} \frac{\omega}{\ell}, \quad \omega=x+i \frac{\mu}{x} .
$$

This applies to $\omega=\omega_{\min }$ and $\omega=\omega_{2}$, proving (3.30).

3.4.1. Solution of the problem for $\theta_{\min } \leq \frac{\pi}{3}$. Introduce the function

$$
\Phi_{L}(\ell)=\mathscr{R}_{L}\left(\ell, x_{\min }\right)-\mathscr{R}_{L}\left(\ell, x_{4}(L, \ell)\right) .
$$

LEMma 3.12. If $\theta_{\min } \leq \frac{\pi}{3}$, there exist $\left(A_{0}, \gamma_{0}\right)$ and $L_{0}=\min \left(\frac{\gamma_{0}}{\sqrt{A_{0}}}, \frac{1}{4 A_{0}^{2}},\left(\frac{\gamma_{0}}{2}\right)^{\frac{4}{3}}, A_{0}^{-\frac{3}{4}}, \frac{\gamma_{0}^{3}}{2}\right)$ such that, for any $L<L_{0}$, for any $\tau \in\left[0, \tan \theta_{\min }\right]$, there exists $\ell=\ell_{x}(1+i \tau) \in \mathscr{A}$ with $(\ell, L) \in \mathscr{B}\left(A_{0}, \gamma_{0}\right)$ and $\Phi_{L}(\ell)=0$. The application

$$
\tau \mapsto \ell_{x} \text { such that } \ell=\ell_{x}(1+i \tau) \text { with } \Phi_{L}(\ell)=0,
$$

is continuous from $\left[0, \tan \theta_{\min }\right]$ into $\mathbb{R}_{+}$, which defines a compact set

$$
\mathcal{C}_{L}=\left\{\ell=\ell_{x}(1+i \tau) \in \mathbb{C}, \text { such that } \Phi_{L}(\ell)=0\right\} .
$$

Furthermore, $\ell$ satisfies asymptotically

$$
\sqrt{2 \ell_{x} L} \sim \operatorname{Re} \frac{\omega_{\min }}{\ell} .
$$


Proof. Fix $\left(A_{0}, \gamma_{0}\right)$ from Proposition 3.11. Consider, for fixed $\tau$, the function $\Psi_{\tau}: \ell_{x} \rightarrow \Phi\left(\ell_{x}(1+i \tau)\right)$. It is a continuously differentiable function.

For $(\ell, L) \in \mathscr{B}\left(A_{0}, \gamma_{0}\right), \Psi_{\tau}$ is strictly increasing. Compute by formulas (3.4) with $V(\ell, \omega)=\frac{\omega}{\ell^{2}-\omega^{2}}$,

$\frac{d}{d \ell_{x}} \mathscr{R}_{L}\left(\ell_{x}, \tau \ell_{x}, x_{\text {min }}\right)=D_{\ell} \mathscr{R}_{L}\left(\ell_{x}, \tau \ell_{x}, x_{\min }\right) \cdot(1, \tau)=\mathscr{R}_{L}\left(\ell_{x}, \tau \ell_{x}, x_{\min }\right) \operatorname{Re}\left(V\left(\ell, \omega_{\min }\right)(1+i \tau)\right)$.

As for the extremal point $x_{4}(L, \ell)$,

$\frac{d}{d \ell_{x}} \mathscr{R}_{L}\left(\ell_{x}, \tau \ell_{x}, x_{4}(L, \ell)\right)=\left(D_{\ell} \mathscr{R}_{L}\left(\ell_{x}, \tau \ell_{x}, x_{4}(L, \ell)\right)+\frac{d}{d x} \mathscr{R}_{L}\left(\ell_{x}, \tau \ell_{x}, x_{4}(L, \ell)\right) D_{\ell} x_{4}(L, \ell)\right) \cdot(1, \tau)$

Since $x_{4}$ is a minimum point for $\mathscr{R}_{L}$, the second term vanishes, and therefore

$$
\frac{d}{d \ell_{x}} \mathscr{R}_{L}\left(\ell_{x}, \tau \ell_{x}, x_{4}(L, \ell)\right)=\mathscr{R}_{L}\left(\ell_{x}, \tau \ell_{x}, x_{4}(L, \ell)\right) \operatorname{Re}(V(\ell, \omega)(1+i \tau)) .
$$

Subtracting these two derivatives yields the derivative of $\Psi_{\tau}$ :

$$
\begin{aligned}
\Psi_{\tau}^{\prime}\left(\ell_{x}\right) & =\mathscr{R}_{L}\left(\ell, x_{\min }\right) \operatorname{Re}\left((1+i \tau) V\left(\ell, \omega_{\min }\right)\right)-\mathscr{R}_{L}\left(\ell, x_{4}(L, \ell)\right) \operatorname{Re}\left((1+i \tau) V\left(\ell, \omega_{4}(L, \ell)\right)\right), \\
& =\frac{1}{\ell_{x}}\left(\mathscr{R}_{L}\left(\ell, x_{\min }\right) \operatorname{Re}\left(\ell V\left(\ell, \omega_{\min }\right)\right)-\mathscr{R}_{L}\left(\ell, x_{4}(L, \ell)\right) \operatorname{Re}\left(\ell V\left(\ell, \omega_{4}(L, \ell)\right)\right)\right) .
\end{aligned}
$$

To evaluate the sign of $\Psi_{\tau}^{\prime}\left(\ell_{x}\right)$, a short computation of $\ell V(\ell, \omega)$ is needed. Define $z=\frac{\ell}{\omega}$, and compute

$$
\operatorname{Re} \ell V(\ell, \omega)=\operatorname{Re} \frac{z}{z^{2}-1}=\operatorname{Re} \frac{z\left(\bar{z}^{2}-1\right)}{\left|z^{2}-1\right|^{2}}=\operatorname{Re} \frac{\bar{z}|z|^{2}-z}{\left|z^{2}-1\right|^{2}}=\frac{(\operatorname{Re} z)\left(|z|^{2}-1\right)}{\left|z^{2}-1\right|^{2}} .
$$

With the assumptions on $A_{0}$ and $\gamma_{0}$,

For $\left.\omega=\omega_{\min }, z=\frac{|\ell|}{\left|\omega_{\min }\right|} e^{i\left(\phi-\theta_{\min }\right)} \cdot \phi-\theta_{\min } \in\right]-\frac{\pi}{2}, 0\left[\right.$, then $\operatorname{Re} z>0$ and $|z|^{2} \geq A_{0}>1$, which implies that $\operatorname{Re} \ell V\left(\ell, \omega_{\min }\right)>0$.

For $\left.\omega=\omega_{4}(L, \ell), \phi-\arg (\omega) \in\right] 0, \frac{\pi}{2}\left[\right.$, hence $\operatorname{Re} z>0$ and $|z|^{2} \sim\left(1+\tau^{2}\right) \frac{L \ell_{x}}{2} \leq$ $\left(1+\tan \theta_{\min }^{2}\right) \frac{\gamma_{0}}{2}<1$, which implies that $\operatorname{Re} \ell V\left(\ell, \omega_{4}(L, \ell)\right)<0$.

Therefore for $(\ell, L) \in \mathscr{B}\left(A_{0}, \gamma_{0}\right)$, for fixed $\tau, \Psi_{\tau}^{\prime}\left(\ell_{x}\right)>0$, and the function $\ell_{x} \mapsto$ $\Phi_{L}\left(\ell_{x}(1+i \tau)\right)$ is strictly increasing.

\section{$\Psi_{\tau}$ vanishes at some point.}

$$
L<\frac{\gamma_{0}}{\sqrt{A_{0}}} \Longrightarrow\left((\ell, L) \in \mathscr{B}\left(A_{0}, \gamma_{0}\right) \Longleftrightarrow \ell \in \mathscr{A} \text { and } \sqrt{A_{0}}<\ell_{x}<\frac{\gamma_{0}}{L}\right) .
$$

For any $\tau \in\left[0, \tan \theta_{\text {min }}\right], \ell=\ell_{x}(1+i \tau) \in \mathscr{A}$, and if $\sqrt{A_{0}}<\ell_{x}<\frac{\gamma_{0}}{L}$ we obtain, using the asymptotics in (3.30),

$$
\mathscr{R}_{L}\left(\ell, x_{\text {min }}\right) \sim 1-4 \operatorname{Re} \frac{\omega_{\text {min }}}{\ell} \sim 1-4 \frac{x_{\min }+\tau y_{\text {min }}}{\ell_{x}\left(1+\tau^{2}\right)}, \quad \mathscr{R}_{L}\left(\ell, x_{4}(L, \ell)\right) \sim 1-4 \sqrt{2 \ell_{x} L},
$$

which gives an asymptotics for $\Psi_{\tau}$ :

$$
\Psi_{\tau}\left(\ell_{x}\right) \sim \frac{4}{\ell_{x}}\left(\sqrt{2 \ell_{x}^{3} L}-d_{\min }\right), \quad d_{\min }=\frac{x_{\min }+\tau y_{\min }}{1+\tau^{2}} .
$$


For $\left(\ell=\ell_{x}(1+i \tau), L\right) \in \mathscr{B}\left(A_{0}, \gamma_{0}\right)$,

$$
\left.\ell_{x} \in\right] \frac{1}{2} L^{-\frac{1}{4}}, 2 L^{-\frac{1}{4}}\left[\Longrightarrow \Phi_{L}(\ell)<0, \quad \ell_{x} \in\right] L^{-\frac{2}{3}},\left(\frac{\sqrt{2}}{L}\right)^{\frac{2}{3}}\left[\Longrightarrow \Phi_{L}(\ell)>0 .\right.
$$

- Choose $\left.\ell_{x} \in\right] \frac{1}{2} L^{-\frac{1}{4}}, 2 L^{-\frac{1}{4}}[$.

Then by the assumptions on $L$ in the theorem, $\ell_{x}^{2}>A_{0}$ and $L \ell_{x}<\gamma_{0}$, which shows that $(L, \ell) \in \mathscr{B}\left(A_{0}, \gamma_{0}\right)$. Furthermore

$$
\sqrt{2 L \ell_{x}^{3}}<\sqrt{16 L^{\frac{1}{4}}}<4\left(\frac{\gamma_{0}}{2}\right)^{\frac{1}{6}}
$$

and

$$
4\left(\frac{\gamma_{0}}{2}\right)^{\frac{1}{6}}<x_{\min } \Longrightarrow \Psi_{\tau}\left(\ell_{x}\right)<0
$$

This is realized if $\gamma_{0}<2\left(\frac{x_{\min }}{4}\right)^{6}$.

- Choose $\left.\ell_{x} \in\right] L^{-\frac{2}{3}},\left(\frac{\sqrt{2}}{L}\right)^{\frac{2}{3}}[$.

Again, by the assumptions on $L$ in the theorem, $\ell_{x}^{2}>A_{0}$ and $L \ell_{x}<\gamma_{0}$, which shows that $(L, \ell) \in \mathscr{B}\left(A_{0}, \gamma_{0}\right)$. Furthermore

$$
\sqrt{2 L \ell_{x}^{3}}>\sqrt{2 L^{-1}}>\sqrt{2 A_{0}^{\frac{3}{4}}}
$$

and

$$
\sqrt{2 A_{0}^{\frac{3}{4}}}>2 x_{\min } \Longrightarrow \Psi_{\tau}\left(\ell_{x}\right)>0 .
$$

This is realized if $A_{0}>2^{\frac{4}{3}}$.

Conclusion of the proof. For fixed $\tau$, the application $\ell_{x} \mapsto \Phi_{L}\left(\ell_{x}(1+i \tau)\right)$ is continuous and strictly increasing on the compact set $\left[\sqrt{A_{0}}, \frac{\gamma_{0}}{L}\right]$. By the result above, there is a unique $\ell_{x}$ such that $\Phi_{L}\left(\ell_{x}(1+i \tau)\right)=0$, and it belongs to $] \frac{1}{2} L^{-\frac{1}{4}},\left(\frac{\sqrt{2}}{L}\right)^{\frac{2}{3}}[. \square$

It remains now to minimize $\mathscr{R}_{L}\left(\ell, x_{\text {min }}\right)$ on the set $\mathcal{C}_{L}$ :

LEMMA 3.13. There exist $\left(A_{0}, \gamma_{0}\right)$ and $L_{0}=\min \left(\frac{\gamma_{0}}{\sqrt{A_{0}}}, \frac{1}{4 A_{0}^{2}},\left(\frac{\gamma_{0}}{2}\right)^{\frac{4}{3}}, A_{0}^{-\frac{3}{4}}, \frac{\gamma_{0}^{3}}{2}\right)$ such that, for any $L<L_{0}$, there exists a minimum point $\hat{\ell}$ of $\mathscr{R}_{L}\left(\ell, x_{\min }\right)$ on the compact set $\mathcal{C}_{L}$ :

$$
\mathscr{R}_{L}\left(\hat{\ell}, x_{\text {min }}\right)=\min _{\ell \in \mathcal{C}_{L}} \mathscr{R}_{L}\left(\ell, x_{\text {min }}\right) .
$$

$(\hat{\ell}, L)$ belongs to $\mathscr{B}\left(L_{0}, \gamma_{0}\right)$, and

$$
\hat{\ell} \sim\left(\frac{\left|\omega_{\min }\right|^{2}}{2 L} \cos \frac{\theta_{\min }}{2}\right)^{\frac{1}{3}} e^{i \frac{\theta_{\min }}{2}} .
$$

Furthermore, with the notations in (3.4),

$$
\exists p \in \mathbb{R}_{-}, V\left(\hat{\ell}, \omega_{\min }\right)=p V\left(\hat{\ell}, \omega_{4}(\hat{\ell})\right), \quad p \sim-2 .
$$

Proof. Since $\mathscr{R}_{L}\left(\ell, x_{\min }\right)$ is continuous, it admits a minimum point $\hat{\ell}$ on the compact set $\mathcal{C}_{L}$. We know by Theorem 2.4 that $\hat{\ell}$ is in the interior of $\mathscr{A}$, therefore in the 
interior of $\mathcal{C}_{L}$. Problem (3.34) is a constrained minimization problem whose associated Lagrangian is given by

$$
\mathscr{L}(\ell, \lambda)=\mathscr{R}_{L}\left(\ell, x_{\min }\right)+\lambda \Phi_{L}(\ell)
$$

A necessary condition for a minimum at point $\hat{\ell}$ is the existence of $\lambda \in \mathbb{R}$ such that the Euler-Lagrange equation $D_{\ell} \mathscr{R}_{L}\left(\hat{\ell}, x_{\min }\right)+\lambda D_{\ell} \Phi_{L}(\hat{\ell})=0$, or equivalently

$$
(1+\lambda) D_{\ell} \mathscr{R}_{L}\left(\hat{\ell}, x_{\min }\right)-\lambda D_{\ell}\left(\mathscr{R}_{L}\left(\ell, x_{4}(L, \ell)\right)\right)(\ell=\hat{\ell})=0 .
$$

By definition of $x_{4}(L, \ell), \partial_{x} \mathscr{R}_{L}\left(\ell, x_{4}(L, \ell)\right)=0$, therefore $D_{\ell}\left(\mathscr{R}_{L}\left(\ell, x_{4}(L, \ell)\right)\right)=$ $D_{\ell} \mathscr{R}_{L}\left(\ell, x_{4}(L, \ell)\right)$ and $(3.37)$ is equivalent to $(1+\lambda) D_{\ell} \mathscr{R}_{L}\left(\hat{\ell}, x_{\min }\right)-\lambda D_{\ell} \mathscr{R}_{L}\left(\hat{\ell}, x_{4}(L, \hat{\ell})\right)=$ 0 . Using the definition of $V$ in (3.4) we find that

$$
V\left(\hat{\ell}, \omega_{\min }\right)=\frac{\lambda}{1+\lambda} V\left(\hat{\ell}, \omega_{4}(\hat{\ell}, L)\right) .
$$

Take the asymptotics in the formula for $V$,

$$
V\left(\hat{\ell}, \omega_{\min }\right) \sim \frac{\omega_{\min }}{\hat{\ell}^{2}}, \quad V\left(\hat{\ell}, \omega_{4}(L, \hat{\ell})\right) \sim-\frac{1}{x_{4}(\hat{\ell}, L)} \sim-\sqrt{\frac{L}{2 \hat{\ell}_{x}}} .
$$

By (3.38), since $V\left(\hat{\ell}, \omega_{4}\right)$ is asymptotically real, $V\left(\hat{\ell}, \omega_{\text {min }}\right)$ must be asymptotically real, which gives

$$
\hat{\phi}:=\operatorname{Arg} \hat{\ell} \sim \frac{1}{2} \operatorname{Arg} \omega_{\min }=\frac{1}{2} \theta_{\min } .
$$

This proves that

$$
\hat{\tau}=\frac{\hat{\ell}_{y}}{\hat{\ell}_{x}}=\tan \hat{\phi} \sim \tan \frac{\theta_{\min }}{2},
$$

which determines the principal part of $\hat{\ell}$ as a function of $\hat{\ell}_{x}$ only:

$$
\hat{\ell} \sim \frac{\hat{\ell}_{x}}{\cos \frac{\theta_{\min }}{2}} e^{i \frac{\theta_{\min }}{2}}
$$

Insert into (3.33) to obtain the principal part of $\hat{\ell}_{x}$ :

$$
\sqrt{2 \hat{\ell}_{x} L} \sim \operatorname{Re} \frac{\omega_{\min }}{\hat{\ell}} \sim \frac{\left|\omega_{\min }\right| \cos ^{2}\left(\frac{\theta_{\min }}{2}\right)}{\hat{\ell}_{x}} \Longrightarrow \hat{\ell}_{x} \sim\left(\frac{\left|\omega_{\min }\right| \cos ^{2}\left(\frac{\theta_{\min }}{2}\right)}{\sqrt{2 L}}\right)^{\frac{2}{3}} .
$$

Compute now

$$
\begin{gathered}
\frac{V\left(\hat{\ell}, \omega_{\min }\right)}{V\left(\hat{\ell}, \omega_{4}(L, \hat{\ell})\right)} \sim-\frac{\omega_{\min }}{\hat{\ell}^{2}} \sqrt{\frac{2 \hat{\ell}_{x}}{L}} \sim-2 \frac{\left|\omega_{\min }\right| \cos ^{2} \frac{\theta_{\min }}{2}}{\sqrt{2 L \hat{\ell}_{x}^{3}}} \sim-2 \frac{\left|\omega_{\min }\right| \cos ^{2} \frac{\theta_{\min }}{2}}{\left|\omega_{\min }\right| \cos ^{2}\left(\frac{\theta_{\min }}{2}\right)}, \\
V\left(\hat{\ell}, \omega_{\min }\right) \sim-2 V\left(\hat{\ell}, \omega_{4}(L, \hat{\ell})\right) .
\end{gathered}
$$


TheOREM 3.14. Suppose $k_{\max }=+\infty$ and $\theta_{\min } \leq \frac{\pi}{3}$. Then there exists $L_{0}>0$ such that, for all $L \leq L_{0}$ the optimal parameter $\ell_{L, \infty}^{*}$ is equal to $\hat{\ell} . \ell_{L, \infty}^{*}$ and the corresponding convergence factor $\delta_{L, \infty}^{*}$ admit the following asymptotic expansions:

$$
\ell_{L, \infty}^{*}=\left(\frac{\left|\omega_{\min }\right|^{2}}{2 L} \cos \frac{\theta_{\min }}{2}\right)^{\frac{1}{3}} e^{i \frac{\theta_{\min }}{2}}+\mathcal{O}(1), \quad \delta_{L, \infty}^{*}=1-2 \sqrt{2 L \operatorname{Re} \ell_{L, \infty}^{*}}+\mathcal{O}\left(L^{\frac{2}{3}}\right) .
$$

The alternation points are $\omega_{\min }$ and $\omega_{4}(\hat{\ell})$, with $\operatorname{Re} \omega_{4}(\hat{\ell}) \sim \sqrt{\frac{2 \operatorname{Re} \ell_{L, \infty}^{*}}{L}}$.

Proof. By the results above, there exist $\left(\gamma_{0}, A_{0}\right)$ and $L_{0}$ such that for $L<L_{0}$, there exists $\hat{\ell}$ where a minimum of $\mathscr{R}$ on $\mathcal{C}_{L}$ occurs. Check the position of $x_{2}(\hat{\ell})$ and $x_{\min }$. Those are the abscissae of $\omega_{2}(\hat{\ell})$ and $\omega_{\min }$, and by the asymptotic formulas above and in Proposition 3.10,

$$
\left.\theta_{\min } \in\right] 0, \frac{\pi}{3}\left[\Longrightarrow \theta_{2}(\hat{\ell}) \sim \frac{\pi}{2}-\frac{\theta_{\min }}{2} \in\right] \frac{\pi}{3}, \frac{\pi}{2}[.
$$

Therefore $\left.\theta_{2}(\hat{\ell}) \in\right] \theta_{\min }, \frac{\pi}{2}\left[\right.$, and since the points $\omega_{\min }$ and $\omega_{2}(\hat{\ell})$ belong to the hyperbola, $t_{2}(\hat{\ell})<t_{\text {min }}$. If $\theta_{\min }=\frac{\pi}{3}$, then $t_{\text {min }}=\mu \tau$, and we need more precision, given by asymptotic formula (3.27), which shows that $t_{2}<t_{\min }$.

Then by Proposition 3.11,

$$
h_{L, \infty}(\ell):=\sup _{x \in\left[x_{\min },+\infty\right)} \delta_{L}(\ell, x)=\max \left(\delta_{L}\left(\ell, x_{\min }\right), \delta_{L}\left(\ell, x_{4}(L, \ell)\right)\right) .
$$

It remains to show that $\hat{\ell}$ is the minimum point for $h_{L, \infty}$. Indeed by $(3.38), V\left(\hat{\ell}, \omega_{\min }\right)=$ $-p V\left(\hat{\ell}, \omega_{4}(L, \hat{\ell})\right)$ with $p$ real positive, and the alternation theorem 3.2 applies.

By the uniqueness theorem 2.9, we deduce that $\hat{\ell}$ is the unique solution $\ell_{L, \infty}^{*}$ of Problem (1.3), which terminates the proof of Theorem 3.14. The asymptotic formula for the convergence factor is now obtained by using formulas (3.30).

3.4.2. Solution of the problem for $\theta_{\min }>\frac{\pi}{3}$. In this case, the local internal maximum points are $x_{2}$ and $x_{4}$. Introduce the function

$$
\Phi_{L}(\ell)=\mathscr{R}_{L}\left(\ell, x_{2}(L, \ell)\right)-\mathscr{R}_{L}\left(\ell, x_{4}(L, \ell)\right)
$$

Lemma 3.15. If $\theta_{\min } \geq \frac{\pi}{3}$, there exist $\left(A_{0}, \gamma_{0}\right)$ and $L_{0}=\min \left(\frac{\gamma_{0}}{\sqrt{A_{0}}}, \frac{1}{4 A_{0}^{2}},\left(\frac{\gamma_{0}}{2}\right)^{\frac{4}{3}}, A_{0}^{-\frac{3}{4}}, \frac{\gamma_{0}^{3}}{2}\right)$ such that, for any $L<L_{0}$, for any $\tau \in\left[0, \tan \theta_{\min }\right]$, there exists $\ell=\ell_{x}(1+i \tau) \in \mathscr{A}$ with $(\ell, L) \in \mathscr{B}\left(A_{0}, \gamma_{0}\right)$ and $\Phi_{L}(\ell)=0$. Furthermore $\ell_{x}$ is a continuous function of $\tau$, and

$$
2 \ell_{x}^{3} L \sim \frac{4 \mu \tau}{\left(1+\tau^{2}\right)^{2}} .
$$

Proof. The proof is identical to that of Lemma 3.12, replacing $\left(\omega_{\min }, \omega_{2}(\ell)\right)$ by $\left(\omega_{2}(\ell), \omega_{4}(\ell)\right)$.

The application $\tau \mapsto \ell_{x}$ such that $\ell=\ell_{x}(1+i \tau)$ with $\Phi_{L}(\ell)=0$, is continuous from $\left[0, \tan \theta_{\text {min }}\right]$ into $\mathbb{R}_{+}$. The set $\mathcal{C}_{L}$ of $\ell=\ell_{x}(1+i \tau) \in \mathbb{C}$ with $\Phi_{L}(\ell)=0$ is compact. 
LEMma 3.16. There exist $\left(A_{0}, \gamma_{0}\right)$ and $L_{0}=\min \left(\frac{\gamma_{0}}{\sqrt{A_{0}}}, \frac{1}{4 A_{0}^{2}},\left(\frac{\gamma_{0}}{2}\right)^{\frac{4}{3}}, A_{0}^{-\frac{3}{4}}, \frac{\gamma_{0}^{3}}{2}\right)$ such that, for any $L<L_{0}$, there exists a minimum point $\hat{\ell}$ of $\mathscr{R}_{L}\left(\ell, x_{2}(\ell)\right)$ on the compact $\operatorname{set} \mathcal{C}_{L}$ :

$$
\mathscr{R}_{L}\left(\hat{\ell}, x_{2}(\hat{\ell})\right)=\min _{\ell \in \mathcal{C}_{L}} \mathscr{R}_{L}\left(\ell, x_{2}(\ell)\right)
$$

It behaves asymptotically as $\hat{\ell} \sim \sqrt[3]{\frac{\mu}{L}} e^{i \frac{\pi}{6}}$, furthermore, with the notations in (3.4),

$$
\exists p \in \mathbb{R}_{-}, \quad V\left(\hat{\ell}, \omega_{2}(\hat{\ell}, L)\right)=p V\left(\hat{\ell}, \omega_{4}(\hat{\ell})\right), \quad p \sim-2 .
$$

Proof. The proof of Lemma 3.13 applies verbatim, until

$$
\exists p \in \mathbb{R}, \quad V\left(\hat{\ell}, \omega_{2}(\hat{\ell}, L)\right)=p V\left(\hat{\ell}, \omega_{4}(\hat{\ell}, L)\right) .
$$

Take the principal parts, using that $(\hat{\ell}, L) \in \mathscr{B}\left(A_{0}, \gamma_{0}\right)$, to obtain

$$
V\left(\hat{\ell}, \omega_{2}(\hat{\ell}, L)\right) \sim \frac{\omega_{2}(\hat{\ell}, L)}{\hat{\ell}^{2}}, \quad V\left(\hat{\ell}, \omega_{4}(L, \hat{\ell})\right) \sim-\frac{1}{x_{4}(\hat{\ell}, L)} \sim-\sqrt{\frac{L}{2 \hat{\ell}_{x}}} \in \mathbb{R} .
$$

Since $V\left(\hat{\ell}, \omega_{4}(\hat{\ell}, L)\right)$ is asymptotically real, $V\left(\hat{\ell}, \omega_{2}(\hat{\ell}, L)\right)$ is asymptotically real as well, which implies that its argument is asymptotically 0 , i.e.

$$
\hat{\phi}:=\operatorname{Arg} \hat{\ell} \sim \frac{1}{2} \operatorname{Arg} \omega_{2}(\hat{\ell}, L)=\frac{1}{2} \theta_{2}(\hat{\ell}, L) \sim \frac{1}{2}\left(\frac{\pi}{2}-\hat{\phi}\right) .
$$

This proves that

$$
\hat{\phi} \sim \frac{\pi}{6}, \text { and } \hat{\tau}=\tan \hat{\phi} \sim \frac{1}{\sqrt{3}},
$$

which determines by (3.42) the principal part of $\hat{\ell}_{x}$ and $\hat{\ell}$ :

$$
\hat{\ell}_{x}^{3} \sim \frac{3 \sqrt{3} \mu}{8 L}, \quad \hat{\ell} \sim \frac{\sqrt{3}}{2} \sqrt[3]{\frac{\mu}{L}}\left(1+\frac{i}{\sqrt{3}}\right)=\sqrt[3]{\frac{\mu}{L}} e^{i \frac{\pi}{6}} .
$$

Compute now

$$
\frac{V\left(\hat{\ell}, \omega_{2}(\hat{\ell}, L)\right)}{V\left(\hat{\ell}, \omega_{4}(L, \hat{\ell})\right)} \sim-\sqrt{1+\frac{1}{\hat{\tau}^{2}}} \sim-2 .
$$

This proves also that

$$
\omega_{2}(\hat{\ell}) \sim \sqrt{\mu \hat{\tau}}\left(1+\frac{i}{\hat{\tau}}\right) \sim 2 \sqrt{\mu \tan \frac{\pi}{6}} e^{i \frac{\pi}{3}}
$$

Theorem 3.17. Suppose $k_{\max }=+\infty$ and $\theta_{\min }>\frac{\pi}{3}$. Then there exists $L_{0}>0$ such that, for all $L \leq L_{0}$ the optimal parameter $\ell_{L, \infty}^{*}$ is equal to $\hat{\ell} . \ell_{L, \infty}^{*}$ and the corresponding convergence factor $\delta_{L, \infty}^{*}$ admit the following asymptotic expansions:

$$
\ell_{L, \infty}^{*}=\sqrt[3]{\frac{\mu}{L}} e^{i \frac{\pi}{6}}+\mathcal{O}(1), \quad \delta_{L, \infty}^{*}=1-2 \sqrt{2 L \operatorname{Re} \ell_{L, \infty}^{*}}+\mathcal{O}\left(L^{\frac{2}{3}}\right) .
$$

The alternation points are the internal points $\omega_{2}\left(\ell_{L, \infty}^{*}\right)$ and $\omega_{4}\left(\ell_{L, \infty}^{*}\right)$, with $\omega_{2}\left(\ell_{L, \infty}^{*}\right) \sim$ $2 \sqrt{\mu \tan \frac{\pi}{6}} e^{i \frac{\pi}{3}}$, and $\operatorname{Re} \omega_{4}\left(\ell_{L, \infty}^{*}\right) \sim \sqrt{\frac{2 \operatorname{Re} \ell_{L, \infty}^{*}}{L}}$. 
Proof. From Lemma 3.13, there exist $\left(A_{0}, \gamma_{0}\right)$ such that, defining

$$
L_{0}=\min \left(\frac{\gamma_{0}}{\sqrt{A_{0}}}, \frac{1}{4 A_{0}^{2}},\left(\frac{\gamma_{0}}{2}\right)^{\frac{4}{3}}, A_{0}^{-\frac{3}{4}}, \frac{\gamma_{0}^{3}}{2}\right)
$$

for any $L<L_{0}$, there exists $\hat{\ell}$ which minimizes

$$
h_{L, \infty}(\ell):=\sup _{x \in\left[x_{\min },+\infty\right)} \delta_{L}(\ell, x)=\max \left(\delta_{L}\left(\ell, x_{2}(\hat{\ell})\right), \delta_{L}\left(\ell, x_{4}(L, \ell)\right)\right),
$$

on $\mathcal{C}_{L}$. Since $V\left(\hat{\ell}, \omega_{\min }\right)=-p V\left(\hat{\ell}, \omega_{4}(L, \hat{\ell})\right)$ with $p$ real positive, Theorem 3.2 applies and shows that the unique solution $\ell_{L, \infty}^{*}$ of Problem (1.3) is $\hat{\ell}$.The formula for the convergence factor is now obtained by using the asymptotic formula $1-4 \sqrt{2 \ell_{x} L}$.

To get the complementary terms $\mathcal{O}$, define $\varepsilon=L^{\frac{1}{3}}$, expand $\ell$ and $s_{4}$ at next order

$$
\ell=r_{0} e^{i \theta_{0}} \varepsilon^{-1}+\mathcal{O}(1), s_{4}=2 r_{0} \cos \theta_{0} \varepsilon^{2}+\mathcal{O}\left(\varepsilon^{3}\right)
$$

and proceed.

Theorem 3.18. There exists $L_{0}>0$ and $C>0$ such that, for all $L \leq L_{0}$ and $k_{\max }>C L^{-\frac{2}{3}}$, the optimal parameter $\ell_{L}^{*}$ is equal to $\ell_{L, \infty}^{*}$.

Proof. Apply Theorem 3.17, and compare $t_{\max }$ and $t_{4}\left(\ell_{L, \infty}^{*}\right)$ :

$$
t_{4}\left(\ell_{L, \infty}^{*}\right) \sim 2 \frac{\operatorname{Re} \ell_{L, \infty}^{*}}{L} \sim 2 C^{\prime} L^{-\frac{4}{3}}
$$

where $C^{\prime}$ depends only on $\mu$ and $\omega_{\min }$. Then if $t_{\max }>t_{4}\left(\ell_{L, \infty}^{*}\right)$, the maximum of $\mathscr{R}\left(\ell_{L, \infty}^{*}, x\right)$ over $\left[x_{\min }, x_{\max }\right]$ is equal to the max over $\left[x_{\min },+\infty\right)$ and the previous analysis applies. Since

$$
x_{\max }^{2}-y_{\max }^{2}=k_{\max }^{2}+\alpha,
$$

choosing $k_{\max }^{2}+\alpha>C^{\prime} L^{-\frac{4}{3}}$ ensures that $t_{\max }>t_{4}\left(\ell_{L, \infty}^{*}\right)$. This can be realized with $k_{\max }>C L^{-\frac{2}{3}}$, with $C>\sqrt{C^{\prime}+\alpha L_{0}^{\frac{2}{3}}}$.

Remark 3.19. In computations, $k_{\max }$ is the highest frequency in the numerical solution. If the domain is discretized with a mesh of size $h$ in each direction, then $k_{\max } \bar{\sim} \frac{\pi}{h}$, and the overlap is a few grid points. Therefore the binding condition in the theorem is fulfilled.

3.5. Quality of the asymptotics. We present in this section an example in dimension $d=2$, with $k_{\min }=\pi, k_{\max }=100 \pi$, which would represent a domain decomposition case, with a length of 1 and 100 grid points in the $y$ direction. For $L \geq$ 0 , the operational value of the best parameter given in the introduction, properties 1.C and 1.D is denoted by $\ell_{L}^{o p}$. The operational convergence factor is defined accordingly.

The complex parameter $\eta$ has a fixed imaginary part defined by $\mu=1$, and the value of $\alpha=\operatorname{Re} \eta$ is modified to cover the three ranges of values of $\theta_{\min }$ identified in the analysis, see property 1.C.

The numerical optimum $\ell_{L}^{t h}$ of the continuous function is evaluated by computing $\sup _{k} \delta_{L}(\ell, k)$ on a very fine grid in $k$, for a range of $\ell$, varying $\operatorname{Re} \ell$ and $\operatorname{Im} \ell$ on a fine grid, and taking the minimum value in the table. Then the functions $k \rightarrow \delta_{L}\left(\ell_{L}^{t h}, k\right)$ and $k \rightarrow \delta_{L}\left(\ell_{L}^{o p}, k\right)$ are plotted on the same picture. In Figure 3.6, the overlap is zero, while in Figure 3.7 the overlap is one grid point, which corresponds to $L=\frac{\pi}{k_{\max }}$.

In the last case, $\theta_{\min } \in\left(\frac{\pi}{3}, \frac{\pi}{2}\right)$, figure (d) zooms on the smallest frequencies to see the equioscillation points better. These figures enhance the good behavior of the operational formulas for the best parameter, even in the asymptotic cases. 


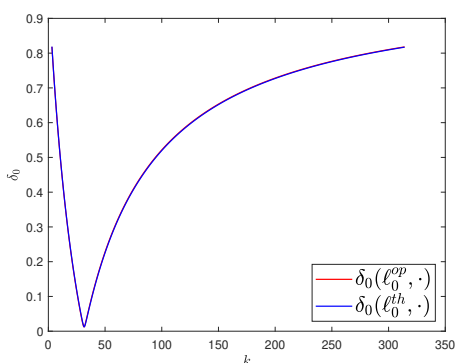

(a) $\theta_{\min } \in\left(0, \frac{\pi}{4}\right)$

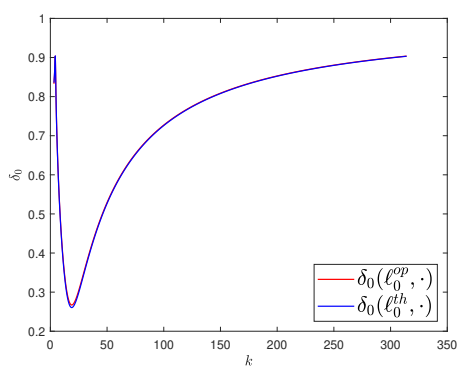

(c) $\theta_{\min } \in\left(\frac{\pi}{3}, \frac{\pi}{2}\right)$

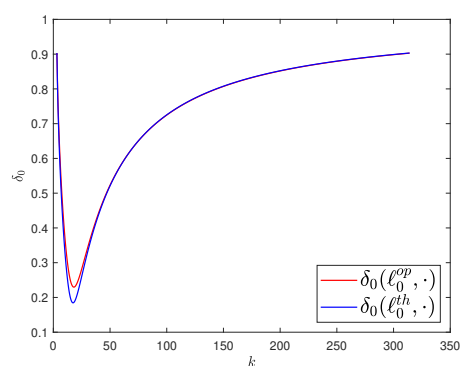

(b) $\theta_{\min } \in\left(\frac{\pi}{4}, \frac{\pi}{3}\right)$

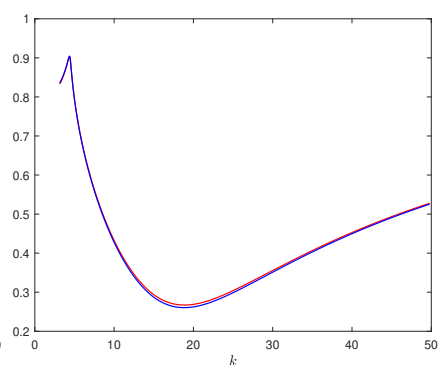

(d) $\theta_{\min } \in\left(\frac{\pi}{3}, \frac{\pi}{2}\right)$, zoom

Fig. 3.6: Plots of $k \mapsto \delta_{0}(\ell, k)$ for $\ell=\ell_{0}^{o p}$ and $\ell=\ell_{0}^{t h}$

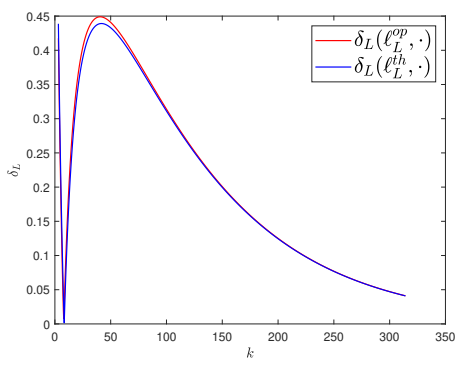

(a) $\theta_{\min } \in\left(0, \frac{\pi}{4}\right)$

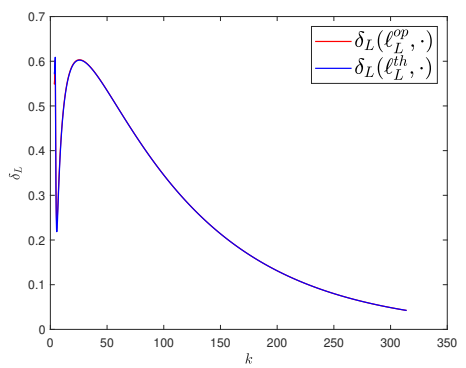

(c) $\theta_{\min } \in\left(\frac{\pi}{3}, \frac{\pi}{2}\right)$

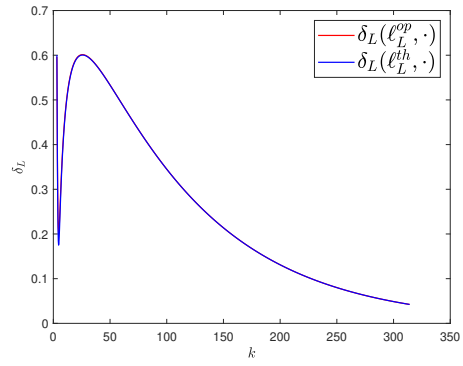

(b) $\theta_{\min } \in\left(\frac{\pi}{4}, \frac{\pi}{3}\right)$

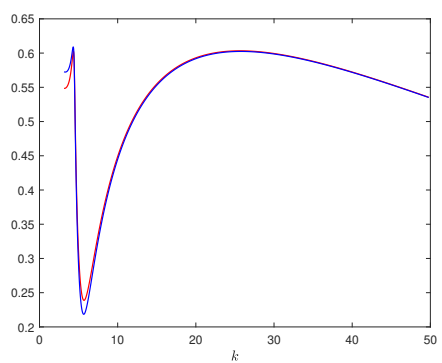

(d) $\theta_{\min } \in\left(\frac{\pi}{3}, \frac{\pi}{2}\right)$, zoom

Fig. 3.7: Graph of $k \mapsto \delta_{L}(\ell, k)$ for $\ell=\ell_{L}^{o p}$ and $\ell=\ell_{L}^{t h}$ 


\section{Application to the optimal control problem.}

4.1. Description of the problem. Consider a conductive body occupying a domain $\Omega \subset \mathbb{R}^{d}$. The temperature is fixed on the boundary, heat sources are represented by a function $f \in L^{2}(\Omega)$, and a control may be provided in a part $\underline{\Omega}$ of $\Omega$, defined by $v \in L^{2}(\underline{\Omega})$. The state of the system is the temperatures field $y$, defined by the Poisson equation

$$
\begin{cases}-\Delta y=f+v & \text { in } \Omega \\ y=0 & \text { on } \partial \Omega .\end{cases}
$$

For a given $v$, the equation above has a unique solution in $H_{0}^{1}(\Omega)$, that will be called $y(v)$ to stress the dependency in $v$. Let $y_{d}$ be a given temperature profile target, the optimal control problem is defined as the minimization of the cost function

$$
J(v)=\frac{1}{2} \int_{\Omega}\left(y(v)-y_{d}\right)^{2} d x+\frac{\nu}{2} \int_{\underline{\Omega}} v^{2} d x .
$$

The first term measures the distance to the desired profile $y_{d}$, and the second term the energy consumption. The weight parameter $\nu$ is defined by the user, corresponding to what effect is to be privileged: a small coefficient $\nu$ means that the user wants to approach the desired state without caring about the cost in energy, while large $\nu$ means to reduce the cost in energy. The functional $J$ is strictly convex and classical optimisation results show that for any $\nu>0$, there is a unique control $u$. The optimal control $u$ and the optimal state $y$ can be computed by introducing the dual state $p \in H_{0}^{1}(\Omega)$, see [30]. In the simplest case of distributed control, that is $\underline{\Omega}=\Omega$, with controls in $H_{0}^{1}(\Omega)$, the optimal control $u$, the optimal state $y$ and the adjoint state $p$ are related by

$$
\left\{\begin{array}{l}
-\Delta y=f+u,\left.y\right|_{\partial \Omega}=0, \\
-\Delta p=y-y_{d},\left.p\right|_{\partial \Omega}=0, \quad p=-\nu u .
\end{array}\right.
$$

Domain decomposition algorithms for this problem have received much attention, see $[8,26,1,31,29,21,39]$. More particularly Benamou in [3] used the newly established non-overlapping domain decomposition algorithm written by Després in [5] for the Helmholtz equation to design a new algorithm for (4.3).

The particular case of distributed control allows for a clever trick, see [3]. Introducing the new unknown $w=y-\frac{i}{\sqrt{\nu}} p$, Problem (4.3) is equivalent to the complex problem: find $w \in H_{0}^{1}(\Omega)$ such that

$$
-\Delta w+\frac{i}{\sqrt{\nu}} w=g \text { in } \Omega \quad \text { with } g=f+\frac{i}{\sqrt{\nu}} y_{d} .
$$

This is a Helmholtz equation with a complex coefficient $\eta=\frac{i}{\sqrt{\nu}} \in i \mathbb{R}$, to which the analysis above applies. In [3], the author proves convergence of the non-overlapping algorithm, and shows that each iterate corresponds to optimal control problems in the subdomains. About the way of choosing the parameter $\ell$, cite [4]: The parameter $\beta$ (here $\ell$ ) has a decisive influence on the speed of convergence. We always chose it proportional to $1 / h$, where $h$ is the size of the finite elements. In this case, the discrete transmission conditions are adimensional. The theoretical analysis in the previous sections clarifies the choice of the optimal parameter under the assumption that $\nu$ is independent of $h$. In that case, the optimal parameter is proportional to $1 / \sqrt{h}$. However, we could recover the results of [4] by extending our analysis to the case $\nu=h^{4}$, see also Section 5.3. 
4.2. Numerical study. We consider here the Helmholtz equation (4.4) in $\Omega=$ $(0,2) \times(0,1)$, discretized with the usual centered second order finite difference scheme. The domain decomposition scripts are adapted from those described in [18]. In a first stage, we analyze the performance of the operational parameter for two subdomains, comparing the convergence with that obtained with a numerical parameter computed by a Neldar-Mead simplex algorithm performed on the numerical error. In a second stage, we compute the control of the heat in a room with various physical boundary conditions, using the parallel algorithm, with three subdomains.

4.2.1. Optimality of the operational parameter. Here we solve the homogeneous equation, that is no internal source $g$ nor boundary source, thus computing the error. The mesh size is the same in the $x$ and $y$ direction, equal to $h=0.01$. Two subdomains of equal size are considered without overlap, or with an overlap of one gridpoint, that is $L=h$.

A numerically best parameter $\ell_{L}^{\text {num }}$ is computed by a Nelder-Mead Simplex Method (Matlab fminsearch) minimizing the solution after 20 iterations, with a uniformly random initial guess.

Then the domain decomposition algorithm is run with a uniformly random initial guess. Figure 4.1 displays in the semilog scale the $L^{\infty}$ error on the interface of the first subdomain, as a function of the iteration number $n$, comparing the convergence behavior over 20 iterations for the classical algorithm and the Robin algorithm, with and without overlap, together with the theoretically expected behavior in dash. As it is well-known in the domain decomposition community, the overlapping

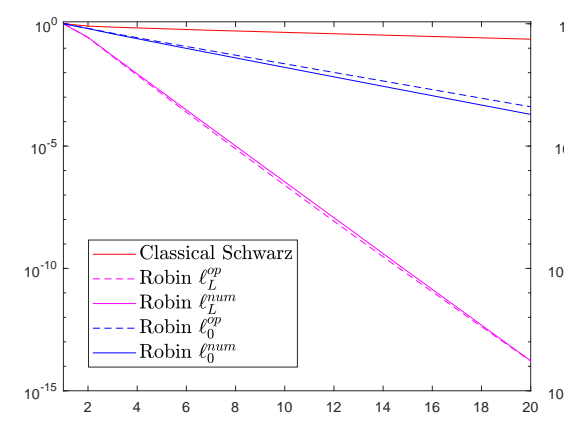

(a) $\nu=1$

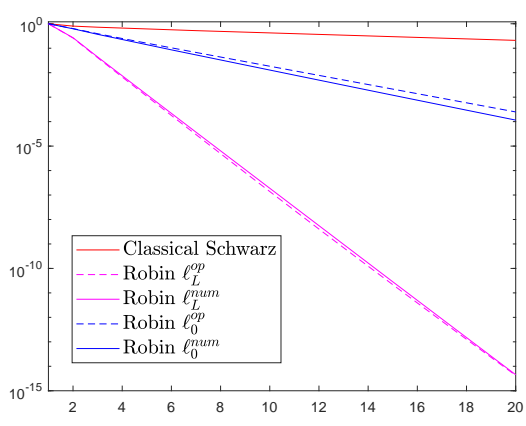

(b) $\nu=0.01$

Fig. 4.1: Convergence history for Classical Schwarz and optimized Robin algorithms

Robin-Schwarz outperforms the non-overlapping Robin-Schwarz which outperforms the classical Schwarz.

Secondly, these plots show that the asymptotic regime for the computation of the coefficients is attained quite rapidly. In the overlapping case for instance, with $L=0.01$, the first term in the asymptotics in $L^{\frac{1}{3}}$ is sufficient to fit the theoretical convergence behavior. Furthermore we see that the convergence properties do not deteriorate when the coefficient $\nu$ decreases.

4.3. Example of optimal control. We describe here a simple example: the control of the temperature in a square room. The room has a fixed temperature on three walls, the western wall communicates with another heated room through a door, 
and the eastern wall is insulated. This example of room has been presented before, for instance in [18]. The radiant floor heating is represented by a distributed control $u$ and $f=0$. The temperature profile target is constant equal to $y_{d}=1$.

The discretizations of the solution $y$ and the control $u$ are represented on Figure 4.2 for values of $\nu$ in the range $(1,0.01,0.001,0.0001)$. As expected, when $\nu$
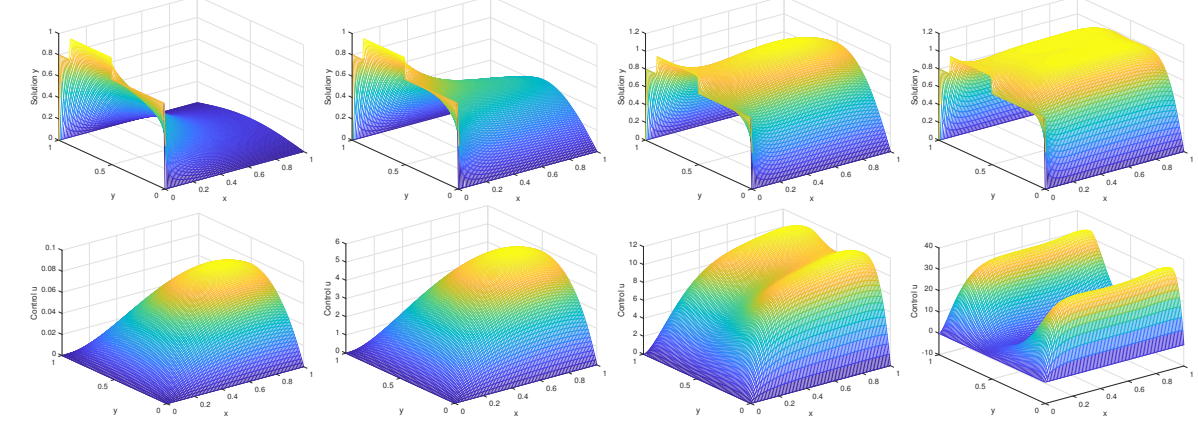

Fig. 4.2: Solution (top) and control (bottom) for four values of the parameter $\nu \in$ $\{1,0.01,0.001,0.0001\}$ starting from $\nu=1$ on the left

decreases, the control $u$ becomes more expansive, but the approximation of the desired solution is better. Furthermore, the control becomes more concentrated along the Dirichlet walls. A computation on a more refined grid has been performed to validate the solution for small values of $\nu$.

We display in Figure 4.3 the iterates 1, 2, 5 and 10 of $y$ for the classical Schwarz algorithm, the overlapping and non-overlapping Robin-Schwarz with the operational parameters described in the analysis. The overlap is kept constant equal to 1 grid points. There are three subdomains of equal size.

In this example, the algorithms are run in parallel. As expected, the order of performances described before is respected, the best performance is reached by overlapping optimized Robin. Even with one gridpoint only in the overlap, the convergence is very fast.

Dirichlet-Neumann and Neumann-Neumann algorithms have been used in connection with optimal control, see $[8,26,25]$. They both use a relaxation parameter. In [21], the authors analyze the convergence factors of the Dirichlet-Neumann Algorithm in one dimension of space. They find that with two subdomains, the DirichletNeumann algorithm converges in two iterations when the subdomains are of equal size and the relaxation parameter is equal to $1 / 2$. Convergence in three iterations appears to be true also for our problem, when three subdomains of equal size are used and the relaxation parameter is equal to $1 / 2$.

The situation is very different when the subdomains are not of equal size. The plots in Figure 4.4 display the iterates when the middle subdomain is much smaller than the others. In the absence of hints on how to compute the relaxation parameter in Dirichlet-Neumann, we chose it to be 0.5 on both interfaces. The parameter $\ell$ for the Robin-Schwarz algorithm is the optimized coefficient.

For a non symmetric decomposition, classical Schwarz still converges, but very slowly, and Robin Schwarz is very robust, especially with overlap. But DirichletNeumann does not converge anymore. No analysis is available in two dimensions, and we believe that our formalism might be useful in that context as well. 

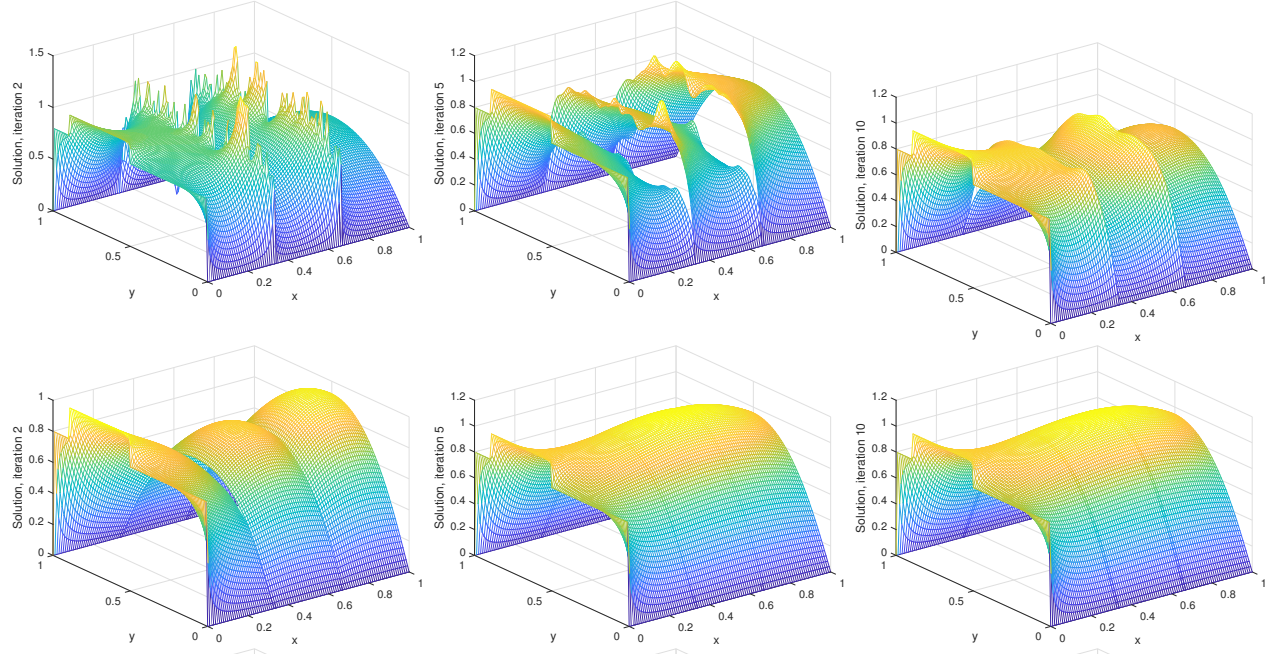

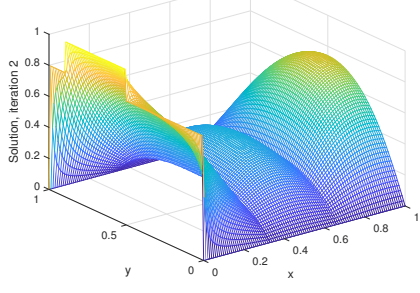

(a) Solution Iteration 2

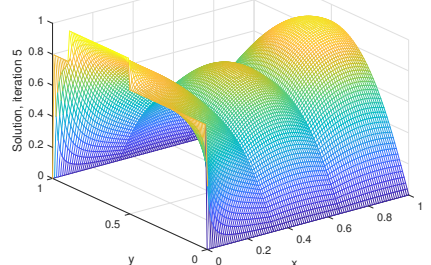

(b) Solution Iteration 5

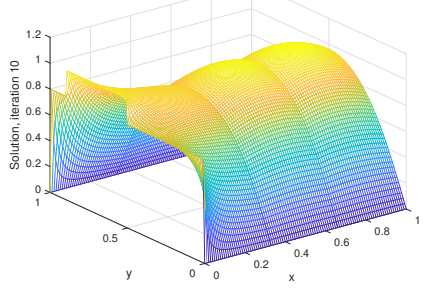

(c) Solution Iteration 10

Fig. 4.3: Iterates of the solution $y$ for classical Schwarz (top row), Overlapping Robin Schwarz (second row), Non-overlapping Robin Schwarz (third row). $\nu=0.001$.

5. Extensions. For the isotropic Laplace operator, we just saw that the operational formulas we obtained perform very well, even with multiple subdomains. The importance of a bounded and discrete analysis appears for anisotropic elliptic real equations. In [19], the analysis of the best approximation problem has been done completely in the non-overlapping case, for two subdomains of equal size. Extension to subdomains of different sizes, still needs to be addressed. We show the formulas for the convergence factors in the next section.

5.1. Continuous and discrete analysis, bounded domain. Suppose that the domain is bounded also in the $x$ direction, that is $\Omega=\left(-c_{1}, c_{2}\right) \times D$. Then it is easy to generalize formulas $(2.5)$ to

$$
\hat{e}_{1}^{n}=a_{1}^{n} \sinh \left(\omega\left(x+c_{1}\right)\right), \quad \hat{e}_{2}^{n}=a_{2}^{n} \sinh \left(\omega\left(c_{2}-x\right)\right) .
$$

For the classical Schwarz algorithm with Dirichlet transmission condition the convergence factor is $\left(\rho=\delta^{2}\right)$

$$
\rho_{L, D}^{c}=\left|\begin{array}{ll}
\frac{\sinh \omega\left(c_{2}-L\right)}{\sinh \omega\left(c_{1}+L\right)} & \frac{\sinh \omega c_{1}}{\sinh \omega c_{2}}
\end{array}\right|,
$$



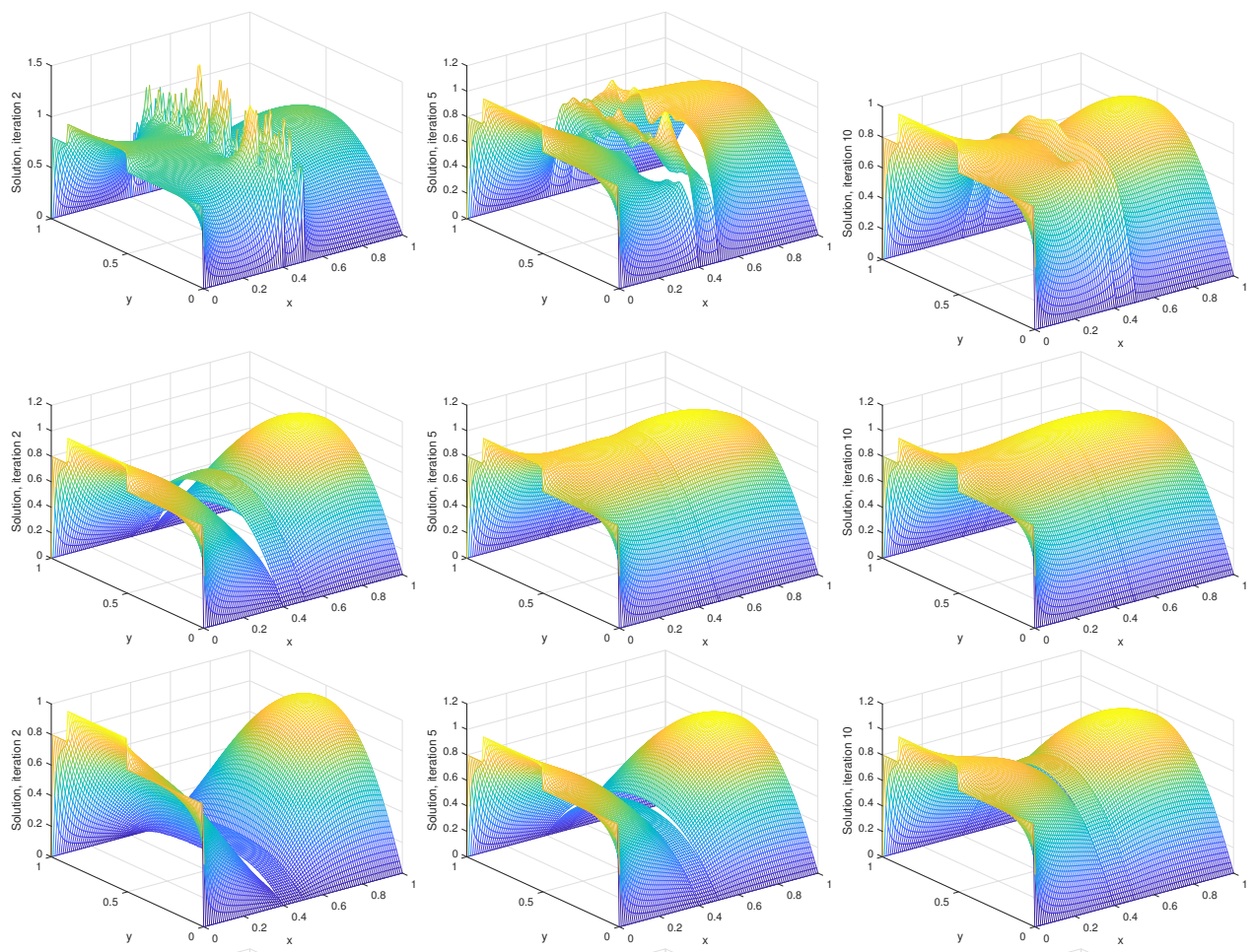

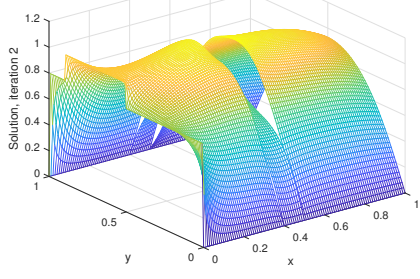

(a) Solution Iteration 2

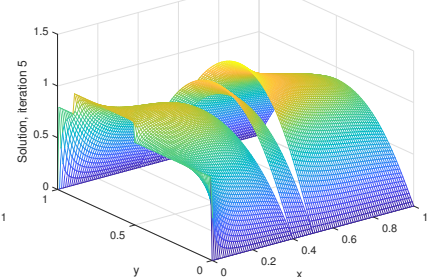

(b) Solution Iteration 5

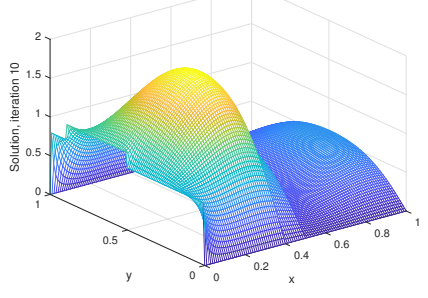

(c) Solution Iteration 10

Fig. 4.4: Solution $y$ for classical Schwarz (top row), Overlapping Robin Schwarz (second row), Non-overlapping Robin Schwarz (third row) and Dirichlet-Neumann (bottom) for $\nu=0.001$.

and for the Robin transmission conditions we obtain the convergence factor

$$
\rho_{L, R}^{c}=\rho_{L, D}^{c} \quad\left|\frac{\ell-\omega \operatorname{coth} \omega\left(c_{2}-L\right)}{\ell+\omega \operatorname{coth} \omega\left(c_{1}+L\right)} \frac{\ell-\omega \operatorname{coth} \omega c_{1}}{\ell+\omega \operatorname{coth} \omega c_{2}}\right| .
$$

Introduce now the classsical second order finite difference scheme, a mesh $\left(h_{x}, h_{y}\right)$, with $M_{j}$ points in $x$ in $\Omega_{j}$, and $p$ points in the overlap. Then the convergence factor can be computed using Fourier series, we extend to the complex case the formulas in [19]. Define

$$
\begin{aligned}
& \alpha(k):=\frac{4}{h_{y}^{2}} \sin ^{2}\left(\frac{k h_{y}}{2}\right), \quad \mu(k):=h_{x}^{2}(\alpha(k)+\eta) \in \mathbb{C} \backslash \mathbb{R}_{-}, \\
& \lambda(k):=1+\frac{\mu(k)}{2}-\sqrt{\mu(k)+\frac{\mu(k)^{2}}{4}} \in D(0,1) \backslash \mathbb{R}_{-}, \nu(k):=\ln \lambda(k),
\end{aligned}
$$


The discrete convergence factor for classical Schwarz is

$$
\rho_{p, D}^{d}=\frac{\sinh \left(\left(M_{2}-p\right) \nu\right)}{\sinh \left(\left(M_{1}+p\right) \nu\right)} \frac{\sinh \left(M_{1} \nu\right)}{\sinh \left(M_{2} \nu\right)}
$$

and for optimized Schwarz, it takes the form

$$
\rho_{p, R}^{d}=\rho_{p, D}^{d} \frac{\ell-\frac{2}{h_{x}} \tanh \frac{\nu}{2} \operatorname{coth}\left(\left(M_{2}-p\right) \nu\right)}{\ell+\frac{2}{h_{x}} \tanh \frac{\nu}{2} \operatorname{coth}\left(\left(M_{1}+p\right) \nu\right)} \frac{\ell-\frac{2}{h_{x}} \tanh \frac{\nu}{2} \operatorname{coth}\left(M_{1} \nu\right)}{\ell+\frac{2}{h_{x}} \tanh \frac{\nu}{2} \operatorname{coth}\left(M_{2} \nu\right)} .
$$

The analysis of the min-max problems related to these convergence factors needs extensions of our strategy. When $c_{2}=c_{1}-L$, they will be rather straightforward. In the other cases, even for real coefficient $\eta$, there is no available results at the moment.

5.2. Extension to Ventcel transmission conditions. In the Ventcel transmission conditions, a higher order part is added in the Robin operator $\partial_{x}+\ell$ as $\partial_{x}+\ell-\tilde{\ell} \Delta_{y}$. In Fourier variables, $\ell$ is replaced by $\ell+\tilde{\ell} k^{2}$. The min-max problem has now two complex unknowns

$$
\inf _{(\ell, \tilde{\ell}) \in \mathbb{C}^{2}} \sup _{k \in K}\left|\frac{\ell+\tilde{\ell} k^{2}-\omega(k)}{\ell+\tilde{\ell} k^{2}+\omega(k)} e^{-L \omega(k)}\right|
$$

which is a homographic best approximation problem in $\mathbf{P}_{1}(\mathbb{C})$. This problem has been analyzed in a real frame, see [16], in the complex case with symmetry where the coefficients are real in [7]. Its resolution needs a new analysis, extending to polynomials of degree $n$ our new alternation property 1.B.

5.3. Particular cases. In the previous analysis in Section $3, \eta$ is a constant complex number. But in several applications, $\eta$ is related to $h$, as described below:

Case 1 In the control problem (4.2), the penalisation parameter $\nu$ might be related to the mesh size $h$ by $\nu \approx h^{2 s}$.

Case 2 For propagation in a circuit with conductivity $\sigma$, of harmonic waves with frequency $\kappa, \alpha=-\kappa^{2}<0$ and $\beta=\sigma \kappa$. In the discretization process, $\kappa$ and $h$ are related by the rule from Shannon's sampling theorem, $\kappa h=\frac{2 \pi}{G}$ where $G$, the number of gridpoints by wavelength, is between 6 and 10 for a good sampling; see [41, 34]. Finite element estimates for $P_{1}$ show that the error is bounded by $c\left(\kappa h+\kappa^{3} h^{2}\right)$, see [27], therefore $\kappa^{3} h^{2}$ must be small as well.

Case 3 Again for wave propagation and $\mu=0$, when using radiation transmission conditions as in Després [12], that is with $\ell=i \kappa$, the convergence factor is equal to 1 for $k=\kappa$. A Robin-Schwarz strategy has been developed, minimizing the convergence factor away from $k_{c}$, and using GMRES algorithm to manage $k_{c}$, see [15]. The so-called shifted laplacian technique is often used, with $\mu<\frac{1}{\kappa}$, see [13].

The analysis described above applies, straightforwardly in the nonoverlapping case, using a scaling with parameter $L$ in the overlapping case. 
Table 5.1: Definitions and references for the objects used in the text

\begin{tabular}{|c|c|c|}
\hline$K, k_{\min }, k_{\max }$ & $(1.2)$ & $K=\left[k_{\min }, k_{\max }\right]$ \\
\hline$\delta_{L}$ & $(1.1)$ & $\delta_{L}(\ell, k)=\left|\frac{\omega(k)-\ell}{\omega(k)+\ell} e^{-L \omega(k)}\right|, \omega(k)=\sqrt{k^{2}+\eta}$ \\
\hline$\omega(k)$ & (1.1)-right & $\omega(k)=\sqrt{k^{2}+\eta}$ \\
\hline$\omega_{\min }, \omega_{\max }$ & $(1.8)$ & $\omega\left(k_{\min }\right), \omega\left(k_{\max }\right)$ \\
\hline$\theta_{\min }, \theta_{\max }$ & $(1.8)$ & $\operatorname{Arg}\left(\omega_{\min }\right), \operatorname{Arg}\left(\omega_{\max }\right)$ \\
\hline$\eta, \alpha, \mu$ & $(2.1)$ & $\eta=\alpha+2 i \mu$ \\
\hline $\mathcal{Q}$ & Theorem 2.1 & $\mathcal{Q}=\{z \in \mathbb{C}, \operatorname{Arg} z \in] 0, \frac{\pi}{2}[\}$ \\
\hline $\mathscr{A}$ & $(2.8)$ & $\mathscr{A}=\left\{z \in \mathbb{C}, \operatorname{Arg} z \in\left[\theta_{\max }, \theta_{\min }\right]\right\}$ \\
\hline$\Gamma$ & Figure 2.1 & $\Gamma=\{\omega(k), k \in K\}$ \\
\hline $\mathcal{C}(\delta)$ & $(2.10)$ & $\mathcal{C}(\delta)=\left\{z \in \mathbb{C},\left|\frac{z-1}{z+1}\right|=\delta\right\}$ \\
\hline $\mathcal{D}(\delta)$ & $(2.10)$ & $\mathcal{D}(\delta)=\left\{z \in \mathbb{C},\left|\frac{z-1}{z+1}\right|<\delta\right\}$ \\
\hline$h_{L}$ & $(2.9)$ & $h_{L}(\ell)=\sup _{k \in K} \delta_{L}(\ell, k)=\sup _{\omega \in \Gamma} \delta_{L}(\ell, \omega)$ \\
\hline $\mathscr{R}_{L}$ & $(3.1)$ & $\mathscr{R}_{L}(\ell, \omega)=\delta_{L}(\ell, \omega)^{2}$ \\
\hline$\ell_{x}, \ell_{y}, \tau$ & $(3.2)$ & $\ell=\ell_{x}+i \ell_{y}, \tau=\frac{\ell_{y}}{\ell_{x}}$ \\
\hline$t, \theta$ & Notation 3.1 & $t=x^{2}, \theta=\arg \omega$ \\
\hline$q_{L}, q_{0}, \tilde{q}$ & Notation 3.1 & $q_{L}(\ell, t)=q_{0}(\ell, t)+L \tilde{q}(\ell, t)$ \\
\hline$V(\ell, \omega)$ & $(3.4)$ & $V(\ell, \omega)=\frac{\omega}{\ell^{2}-\omega^{2}}$ \\
\hline$Q(\ell, \omega, Z)$ & $(3.4)$ & $Q(\ell, \omega, Z)=|Z|^{2}-\operatorname{Re}\left(\frac{\ell-\omega}{\omega} Z^{2}\right)$ \\
\hline $\mathscr{B}(A, \gamma)$ & $(3.24)$ & $\mathscr{B}(A, \gamma)=\left\{(\ell, L) \in \mathcal{A} \times \mathbb{R}_{+}^{*}, A<\ell_{x}^{2}\right.$ and $\left.L \ell_{x}<\gamma\right\}$ \\
\hline
\end{tabular}

REFERENCES

[1] R. A. Bartlett, M. Heinkenschloss, D. Ridzal, and B. G. van Bloemen Waanders, Domain decomposition methods for advection dominated linear-quadratic elliptic optimal control problems, Comput. Methods Appl. Mech. Engrg., 195 (2006), pp. 6428-6447.

[2] A. Bayliss AND E. Turkel, Radiation boundary conditions for wave-like equations, Comm. Pure and Appl. Math., 33 (1980), pp. 707-725.

[3] J.-D. Benamou, A domain decomposition method with coupled transmission conditions for the optimal control of systems governed by elliptic partial differential equations, SIAM J. Numer. Anal., 33 (1996), pp. 2401-2416.

[4] J. D. Benamou, Domain decomposition, optimal control of systems governed by partial differential equations, and synthesis of feedback laws, J. Optim. Theory Appl., 102 (1999), pp. $15-36$.

[5] J.-D. Benamou And B. Després, A domain decomposition method for the Helmholtz equation 
and related optimal control problems, J. Comput. Phys., 136 (1997), pp. 68-82.

[6] D. Bennequin, M. Gander, L. Gounin, and L. Halpern, Optimized Schwarz waveform relaxation for advection reaction diffusion equations in two dimensions, Numer. Math., 73 (2016), pp. 167-195.

[7] D. Bennequin, M. J. Gander, and L. Halpern, A homographic best approximation problem with application to optimized Schwarz waveform relaxation, Mathematics of Computation, 78 (2009), pp. 185-223.

[8] A. Bensoussan, R. Glowinski, and J.-L. Lions, Méthode de décomposition appliquée au contrôle optimal de systèmes distribués, in IFIP Technical Conference on Optimization Techniques, Springer, 1973, pp. 141-151.

[9] F. Caetano, M. J. Gander, L. Halpern, J. Szeftel, et Al., Schwarz waveform relaxation algorithms for semilinear reaction-diffusion equations., NHM, 5 (2010), pp. 487-505.

[10] . Cardano, Girolamo, Ars magna or the rules of algebra, Dover Publications, 1968.

[11] B. Delourme, L. Halpern, and B. T. Nguyen, Optimized Schwarz methods for elliptic optimal control problems, in International Conference on Domain Decomposition Methods, Springer, 2017, pp. 215-222.

[12] B. Després, Méthodes de décomposition de domaine pour les problèmes de propagation d'ondes en régimes harmoniques, PhD thesis, Université Dauphine - Paris IX, 1991.

[13] O. ERnst And M. J. Gander, Why it is difficult to solve Helmholtz problems with classical iterative methods, Numer. Algorithms, 69 (2015), pp. 109-144.

[14] M. Gander, L. Halpern, and F. Nataf, Optimized Schwarz methods, in Domain decomposition methods in sciences and engineering XII Chiba, 1999, T. Chan, T. Kako, H. Kawarada, and O. Pironneau, eds., ddm.org, 2001, pp. 15-27.

[15] M. Gander, F. Magoulès, And F. NataF, Optimized Schwarz methods without overlap for the Helmholtz equation, SIAM J. Sci. Comput., 24 (2002), pp. 38-60.

[16] M. J. Gander, Optimized Schwarz Method, SIAM Journal on Numerical Analysis, 44 (2006), pp. 699-731.

[17] M. J. Gander and O. Dubois, Optimized Schwarz methods for a diffusion problem with discontinuous coefficient, Numer. Algorithms, 69 (2015), pp. 109-144.

[18] M. J. Gander and L. Halpern, Méthodes de décomposition de domaines. notions de base, Techniques de l'ingénieur Analyse numérique des équations différentielles et aux dérivées partielles, base documentaire : 42620210. (2012).

[19] M. J. Gander, L. Halpern, F. Hubert, and S. Krell, Discrete optimization of Robin transmission conditions for anisotropic diffusion with discrete duality finite volume methods. 2020, https://hal.archives-ouvertes.fr/hal-02539124.

[20] M. J. Gander, L. Halpern, And F. Magoulès, An optimized Schwarz method with two-sided Robin transmission conditions for the Helmholtz equation, Internat. J. Numer. Methods Fluids, 55 (2007), pp. 163-175.

[21] M. J. GANDER, F. KWOK, AND B. C. MANDAL, Convergence of substructuring methods for elliptic optimal control problems, in Domain decomposition methods in science and engineering, P. Bjørstad, S. Brenner, L. Halpern, H. Kim, R. Kornhuber, T. Rahman, and O. Widlund, eds., vol. 125 of Lect. Notes Comput. Sci. Eng., Springer, Berlin, 2018, pp. 291-300.

[22] T. Hagström, R. P. Tewarson, and A. Jazcilevich, Numerical experiments on a domain decomposition algorithm for nonlinear elliptic boundary value problems., Appl. Math. Lett., 1 (1988), pp. 299-302.

[23] L. Halpern, C. Japhet, And J. Szeftel, Optimized Schwarz waveform relaxation and discontinuous Galerkin time stepping for heterogeneous problems, SIAM J. Numer. Anal., 50 (2012), pp. 2588-2611.

[24] L. Halpern AND J. Szeftel, Optimized and quasi-optimal Schwarz waveform relaxation for the one-dimensional Schrödinger equation, Math. Models Methods Appl. Sci., 20 (2010), pp. 2167-2199.

[25] M. Heinkenschloss And M. Herty, A spatial domain decomposition method for parabolic optimal control problems, Journal of Computational and Applied Mathematics, 201 (2007), pp. $88-111$.

[26] M. Heinkenschloss And H. NGuyen, Balancing Neumann-Neumann methods for elliptic optimal control problems, in Domain decomposition methods in science and engineering, vol. 40 of Lect. Notes Comput. Sci. Eng., Springer, Berlin, 2005, pp. 589-596.

[27] F. Ihlenburg, I. Babuska, and S. Sauter, Reliability of finite element methods for the numerical computation of waves, Advances in Engineering Software, 28 (1997), pp. 417424.

[28] C. JАPHET, Méthodes de décomposition de domaine et conditions aux limites artificielles en mécanique des fluides: méthode optimisée d'ordre 2, PhD thesis, Université Paris Nord - 
Paris XIII, 1998.

[29] J. E. Lagnese And G. Leugering, Domain decomposition methods in optimal control of partial differential equations, vol. 148 of International Series of Numerical Mathematics, Birkhäuser Verlag, Basel, 2004.

[30] J. L. Lions, Optimal control of systems governed by partial differential equations., vol. 170, Springer, Berlin, 1971.

[31] J.-L. Lions and O. Pironneau, Sur le contrôle parallèle des systèmes distribués, C. R. Acad. Sci. Paris Sér. I Math., 327 (1998), pp. 993-998.

[32] P.-L. Lions, On the Schwarz alternating method. III: a variant for nonoverlapping subdomains, in Third Int. Symposium on Domain Decomposition Methods for Partial Differential Equations, T. Chan, R. Glowinski, J. Périaux, and O. Widlund, eds., Philadelphia, PA, 1990, SIAM.

[33] F. Magoulès, D. B. Szyld, And C. Venet, Asynchronous optimized Schwarz methods with and without overlap, Numer. Math., 137 (2017), pp. 199-227.

[34] S. Marburg, Discretization requirements: How many elements per wavelength are necessary?, Computational Acoustics of Noise Propagation in Fluids -Finite and Boundary Element Methods, (2008).

[35] G. Meinardus, Approximation of functions: Theory and numerical methods, vol. 13, Springer Science \& Business Media, 2012.

[36] F. Nataf, F. Rogier, And E. De Sturler, Optimal Interface Conditions for Domain Decomposition Methods, Technical Report 301, CMAP Ecole Polytechnique, 1994, https: //hal.archives-ouvertes.fr/hal-02194208.

[37] T. Rivlin And H. Shapiro, Some uniqueness problems in approximation theory, Communications on Pure and Applied Mathematics, 13 (1960), pp. 35-47.

[38] W. Rudin, Real and complex analysis, Tata McGraw-Hill education, 2006.

[39] A. Schiela AND S. Ulbrich, Operator preconditioning for a class of inequality constrained optimal control problems, SIAM J. Optim., 24 (2014), pp. 435-466.

[40] H. A. Schwarz, Über einen Grenzübergang durch alternierendes Verfahren, Vierteljahrsschrift der Naturforschenden Gesellschaft in Zürich, 15 (1870), pp. 272-286.

[41] C. E. Shannon, A mathematical theory of communication, Bell System Technical Journal, 27 (1948), pp. 379-423, https://doi.org/10.1002/j.1538-7305.1948.tb01338.x, https: //onlinelibrary.wiley.com/doi/abs/10.1002/j.1538-7305.1948.tb01338.x, https://arxiv.org/ abs/https://onlinelibrary.wiley.com/doi/pdf/10.1002/j.1538-7305.1948.tb01338.x.

[42] G. S. Smirnov And R. G. Smirnov, Best uniform approximation of complex-valued functions by generalized polynomials having restricted ranges, J. Approx. Theory, 100 (1999), pp. 284-303.

[43] L. TonelLi, I polinomi d'approssimazione di Tchebychev, Annali di Matematica Pura ed Applicata (1898-1922), 15 (1908), pp. 47-119.

[44] Y. XU AND X. Chen, Optimized Schwarz methods for the optimal control of systems governed by elliptic partial differential equations, Journal of Scientific Computing., 79 (2019), pp. 11821213. 